SYNCHRONIZATION OF MECHANICAL OSCILLATORS:

AN EXPERIMENTAL STUDY

\author{
A Thesis \\ by \\ ROOZBEH DANESHVAR
}

\begin{abstract}
Submitted to the Office of Graduate Studies of Texas A\&M University in partial fulfillment of the requirements for the degree of

MASTER OF SCIENCE
\end{abstract}

December 2010

Major Subject: Electrical Engineering 


\title{
SYNCHRONIZATION OF MECHANICAL OSCILLATORS: \\ AN EXPERIMENTAL STUDY
}

\author{
A Thesis \\ by \\ ROOZBEH DANESHVAR
}

\author{
Submitted to the Office of Graduate Studies of \\ Texas A\&M University \\ in partial fulfillment of the requirements for the degree of \\ MASTER OF SCIENCE
}

Approved by:

Chair of Committee, Tamás Kalmár-Nagy

Committee Members, Takis Zourntos

Deepa Kundur

Karen Butler-Purry

Head of Department, Costas N. Georghiades

December 2010

Major Subject: Electrical Engineering 


\author{
ABSTRACT \\ Synchronization of Mechanical Oscillators: \\ An Experimental Study. (December 2010) \\ Roozbeh Daneshvar, B.S., University of Tehran; \\ M.S., University of Tehran \\ Chair of Advisory Committee: Dr. Tamás Kalmár-Nagy
}

In this research we consider synchronization of oscillators. We use mechanical metronomes that are coupled through a mechanical medium. We investigate the problem for three different cases: 1) In passive coupling of two oscillators, the coupling medium is a one degree of freedom passive mechanical basis. The analysis of the system is supported by simulations of the proposed model and experimental results. 2) In another case, the oscillator is forced by an external input while the input is also affected by the oscillator. This feedback loop introduces dynamics to the whole system. For realization, we place the mechanical metronome on a one degree of freedom moving base. The movements of the base are a function of a feedback from the phase of the metronome. We study a family of functions for the reactions of the base and their impact on the behavior of the metronome. 3) We consider two metronomes located on a moving base. In this case the two metronomes oscillate and as the base is not freely moving, they are not directly coupled to each other. Now based on the feedbacks from the vision system, the base moves and hence the phases of the metronomes are affected by these movements. We study the space of possibilities for the movements of the base and consider impacts of the base movement on the synchronization of metronomes. We also show how such a system evolves in time. 
To all the people who have helped me for this research 


\section{ACKNOWLEDGMENTS}

Research with Dr. Kalmár-Nagy (or Dr. T, as we call him) had many characteristics in common with my thesis research. Our research and our interactions lie at the edge of chaos, i.e. we had our maximum computational capabilities. This edge of chaos is not necessarily a boundary, but a special region. This is the place where you can find systems with lifelike complex behaviors. The research was fruitful and yet full of joy. Despite being tough and stressful filled with frustrations, I had my best year in Texas A\&M University. This was exactly what I expect from a research: when it entered the ordered regime, it adapted itself towards chaos and when it entered the chaotic regime, it avoided chaos and returned to the same onset again. In essence, it was an honor for me to be Dr. T's student. I learned a lot from him, not only how to do good research, but also how to enjoy the research without reducing the high levels of standards ${ }^{1}$. Working with Dr. T was a new phase in my academic life and the first impact is that I have started cranking the wheel ${ }^{2}$ differently.

The first inspirations of parts of this research started with Dr. Alfred Hübler's Nonlinear Dynamics lab in Santa Fe. He showed us demonstrations of "Edge of Chaos" and later he gave me computer codes for simulating this phenomenon with logistic map. Months later I was curious whether I can replicate the same concept in synchronization of metronomes. I am grateful for his inspiring labs; the inspiration has continued so far.

Knowing Dr. Takis Zourntos was a great opportunity for me. I am always thankful for his welcoming and warm attitude which meant a lot to me when I first

\footnotetext{
${ }^{1}$ He has high (and sometimes hard to satisfy) standards and I never saw him to give up any of them, in any occasion.

${ }^{2}$ This is an expression he always uses and made its way as a saying in the research.
} 
joined his research group. We used to have research discussions and he taught me how to think freely and how not to ignore the very ordinary things around myself. Takis also showed me some more human sides of research and how I need to remember that in any research, I am dealing with people after all. Tracey Neal Thompson was a hand who was always ready to help. He was quick and his promptness was a key factor for us to extend our imaginations and the relevant experimental settings were ready in a short time. The experiments of this research have not been possible without his help. Reza Ghasemi has already spent hours of discussion for this research and he is always ready to spend even more hours. I owe many of the contributions of this research to our talks which gave me new insights to the same problem. Ali-Akbar AghaMohammadi has helped me a lot for this research. He has been a versatile source of help and I knew that whenever I am stuck, I can refer to him to find some clues. Yliang Xu has helped me with the image processing sections and I am thankful to him. Soroush Rostami, a true friend since decades ago, was an always present source of help. Mehdi Maadooliat kindly helped me with statistics, Ramin Mehran helped me with image processing and Miyuki Nakajima was an always present support for the last year. I am grateful to all of these people, my mother and my brother whom I have always had the support and many more who have not been mentioned.

And the last but not the least is Elham, my wife. She was the one who was truly with me all the times. The time I was extensively working on my thesis imposed many pressures on our family and she patiently took all of that. One side of living on the edge of chaos is computational capability and lifelike behavior. But it had another tough side and that is the side which she had to tolerate. I am truly grateful to her for all her help and support which has always been there. 


\section{TABLE OF CONTENTS}

CHAPTER

INTRODUCTION . . . . . . . . . . . . . . . . . . . 1

A. Oscillation .................... . . 1

B. Coupled Oscillators . . . . . . . . . . . . . 1

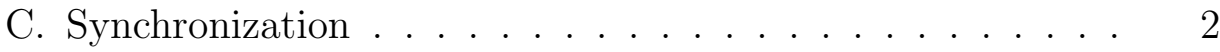

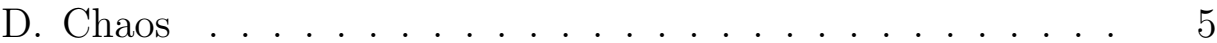

E. Self-Adjusting Systems and the Edge of Chaos . . . . . . 5

F. Purpose of this Research . . . . . . . . . . . . 6

II EXPERIMENTAL SETTINGS . . . . . . . . . . 7

A. Description ................... 7

B. Settings . . . . . . . . . . . . . . 8

C. Image Processing . . . . . . . . . . . . . . . . . 9

1. Finding the Angle . . . . . . . . . . . . . . 11

2. Image Processing Calibration . . . . . . . . . . . . . . 12

3. Validating the Results . . . . . . . . . . . . . 13

D. Active Cart . . . . . . . . . . . . . . . . . 17

E. Experimental Results . . . . . . . . . . . . . . 18

1. Storing the Images . . . . . . . . . . . . . . . . 22

2. Pivot Point . . . . . . . . . . . . . . 23

3. Using Hue-Saturation-Value for Tracking Colors . . . 25

F. Base Setups . . . . . . . . . . . . . . . . . 25

G. Conclusions . . . . . . . . . . . . . 25

III SYNCHRONIZATION . . . . . . . . . . . . . . 27

A. Model of Oscillators . . . . . . . . . . . . . . . . 27

B. Oscillators on a Passive Base . . . . . . . . . . . . . . 30

C. Synchronization on a Passive Base . . . . . . . . . . . . 33

D. Conclusions . . . . . . . . . . . . . . . 38

IV FORCED OSCILLATORS AND ADAPTATION . . . . . . . . 40

A. Forced Oscillators . . . . . . . . . . . . . . . . 40

B. Two Oscillators on an Active Base . . . . . . . . . . . . 41

1. Case 1: $h\left(x_{1}, x_{2}, x_{3}, x_{4}\right)=m \sin \left(x_{2}+x_{4}\right) \ldots \ldots$ 
2. Case 2: $h\left(x_{1}, x_{2}, x_{3}, x_{4}\right)=m \sin \left(x_{1}+x_{3}\right) \ldots \ldots$ C. Two Oscillators on an Active Base with Adjusting Parameter 48 D. Low-Pass Filtered Feedback . . . . . . . . . . . . . 51

E. Adaptation in Experiments . . . . . . . . . . . . . 54

F. Conclusions . . . . . . . . . . . . . . . . 54

V CONCLUSIONS AND FUTURE WORK . . . . . . . . . 58

A. Conclusions . . . . . . . . . . . . . . . . 58

B. Future Work . . . . . . . . . . . . . . . . . 58

1. Model . . . . . . . . . . . . . . . . . . 58

2. Experiments . . . . . . . . . . . . 59

3. Coupling ................... 59

4. Oscillators .................. . . 59

5. Adaptation ................ 60

REFERENCES . . . . . . . . . . . . . . . . . . . 61

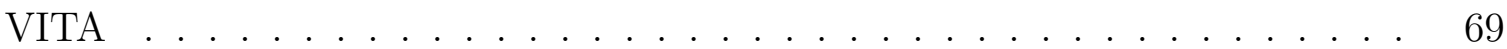




\section{LIST OF FIGURES}

FIGURE

The schematic of the system . . . . . . . . . . . . . 7

2 Experimental settings . . . . . . . . . . . . . . 8

3 Two metronomes on two connected carts . . . . . . . . . . . 11

4 A short time frame of the values from investigation of a metronome for different stickers . . . . . . . . . . . . . . . . . 12

5 The values from investigation of a metronome to show the positions of the stickers . . . . . . . . . . . . . 15

6 The settings and the errors for calibration test . . . . . . . . . 16

7 The position of the cart in a test . . . . . . . . . . . 17

8 The phase of a metronome in time showing the transient region . . 18

9 A short time frame of the metronome phase showing that the shape of the wave is not completely a sine wave . . . . . . . . . . . 19

10 The phase of a metronome in time when the metronome is located on a solid base .................... 20

11 A short time frame of the metronome phase showing sample points . 21

12 A short time frame of the metronome phase to show more details with sample points . . . . . . . . . . . . . . . . 22

13 The distance of the detected pivot point from the regression line of all the stickers for higher frequency . . . . . . . . . . . . 23

14 The distance of the detected pivot point from the regression line of all the stickers for lower frequency . . . . . . . . . . . . . . 24

15 The trajectory of the phase when the base does not move with $\epsilon=0$ 
FIGURE

16 The phase when the base does not move with $\epsilon \neq 0 \ldots \ldots$

17 The schematic of the system for two metronomes located on a passive base . . . . . . . . . . . . . . . . . . . .

18 The Lissajous figure for the coupling parameter values from 0.056 to $0.080 \ldots \ldots \ldots \ldots \ldots \ldots . \ldots \ldots . \ldots \ldots$

19 Phase difference of two metronomes on a passive base $f_{1}=f_{2}=0.4 \mathrm{~Hz}$

20 Phase difference of two metronomes on a passive base $f_{1}=f_{2}=0.6 \mathrm{~Hz} \quad 34$

21 Phase difference of two metronomes on a passive base $f_{1}=f_{2}=0.9 \mathrm{~Hz} \quad 34$

22 Phase difference of two metronomes on a passive base $f_{1}=f_{2}=1.05 \mathrm{~Hz} 35$

23 Phase difference of two metronomes on a passive base $f_{1}=f_{2} \approx 1.27 \mathrm{~Hz} 35$

24 Phase difference of two metronomes on a passive base $f_{1}=f_{2} \approx 1.47 \mathrm{~Hz} 36$

25 Phase difference of two metronomes on a passive base $f_{1}=f_{2}=1.6 \mathrm{~Hz} \quad 36$

26 Phase difference of two metronomes on a passive base $f_{1}=f_{2} \approx 1.73 \mathrm{~Hz} 37$

27 The distances of the detected pivot points from the regression lines of all the stickers . . . . . . . . . . . . . . . . . . 38

28 The trajectory of the phase when the base moves with $\epsilon=0 \ldots 42$

29 The trajectory of the phase when the base moves with $\epsilon \neq 0 \ldots 43$

30 Two metronomes on an actively moving base in which $\dot{x}_{7}=($ ratio $) \theta_{1}+$ $(1-$ ratio $) \theta_{2} \ldots \ldots \ldots \ldots \ldots$

31 Value of $x_{9}$ (the adjustable parameter) in time for initial values from 0.00 to $0.45 \ldots \ldots \ldots$. . . . . . . . . . . . . 52

32 Value of $x_{9}$ (the adjustable parameter) in time for initial value $=0.50 . \quad 53$

33 The Lissajous figures of phases of metronomes in experiment . . . . 55 
FIGURE

34 The Lissajous figures of phases of metronomes through time in

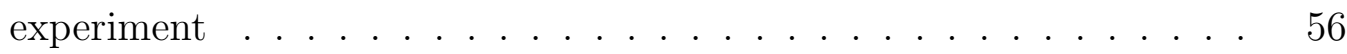

35 The phase difference of metronomes $x_{1}-x_{3}$ and the value of the adjustable parameter $x_{9}$ through time during experiments . . . . . 


\section{CHAPTER I}

\section{INTRODUCTION}

Synchronization is a ubiquitous phenomenon everywhere and it plays an important role in many other phenomena. We start by reviewing some literature about synchronization and some related areas ${ }^{1}$.

\section{A. Oscillation}

The concept of self-oscillations was first proposed by Andronov, Khaikin and Vitt in 1937 [1] (for the English version see [2]). It is mentioned that "A self-oscillating system is an apparatus which produces a periodic process at the expense of a nonperiodic source of energy." Appleton [3] and van der Pol [4,5] set experiments with electric circuits while they were studying the reception of radio signals with electric circuits with triodes. In a relaxed self-sustained oscillator, although the motion of a point in the phase plane might be non-uniform, but the growth of the phase in time is still uniform [6].

\section{B. Coupled Oscillators}

The interaction between the organ pipes was studied by Rayleigh [7]. His experiment involved two organ pipes which had close peach and were located close to each other. The pipes together sounded in perfect unison. Another investigation is done by [8] in which one pipe was substituted by an electric speaker. The authors observed that even minute driving signals forced the pipe to synchronization. A simple model of coupled oscillators is given by [9]:

\footnotetext{
${ }^{1}$ This thesis follows the style of IEEE Transactions on Automatic Control.
} 


$$
\begin{aligned}
& \dot{\theta_{1}}=\omega_{1}+K_{1} \sin \left(\theta_{2}-\theta_{1}\right) \\
& \dot{\theta_{2}}=\omega_{2}+K_{2} \sin \left(\theta_{1}-\theta_{2}\right)
\end{aligned}
$$

in which $\theta_{1}$ and $\theta_{2}$ are the phases of the oscillators and $\omega_{1}$ and $\omega_{2}$ are the natural frequencies. The two parameters $K_{1}$ and $K_{2}$ determine the amount of dependency between the two oscillators (and show that how much they are affected from the other one). For an uncoupled system, we have $K_{1}=K_{2}=0$ and hence the equations are reduced to

$$
\begin{aligned}
& \dot{\theta_{1}}=\omega_{1} \\
& \dot{\theta_{2}}=\omega_{2}
\end{aligned}
$$

\section{Synchronization}

The word synchronous originates from the Greek words chronos and syn which means "sharing the same time" [6]. The authors have considered synchronization as a complex dynamical system rather than a state. Synchronization is thoroughly discussed in [10]. When the oscillators are coupled, there is a phase difference $\phi$ between the two oscillators

$$
\phi=\theta_{1}-\theta_{2}
$$

So, we have 


$$
\begin{aligned}
\dot{\phi} & =\dot{\theta_{2}}-\dot{\theta_{1}} \\
& =\omega_{1}-\omega_{2}-\left(K_{1}+K_{2}\right) \sin \phi
\end{aligned}
$$

in which $\omega_{1}-\omega_{2}$ is also called Frequency detuning [6]. Synchronization can result from an interaction between systems [11] or sub-systems [12-14].

Synchronization of two coupled escapement-driven pendulum clocks was investigated in [15]. Blekhman [16] discusses observations of the Dutch researcher Christian Huygens $^{2}$ and presents the results of a laboratory reproduction and a theoretical analysis of oscillators coupled through a common supporting frame. At first, Huygens suspected the "sympathy" between the clocks was due to induced air currents, but eventually concluded that the cause was the "imperceptible movements" of the common supporting structure [17]. In the reproduction of Huygens experiment [17], the anti-phase state was the only type of synchronization that was observed.

The first observations of synchronization in electronic tube generators were done by Eccles $[18,19]$. He considered the problem of creating a precision clock and the transmission of naval signals. In [20] the authors experimentally studied rhythmic hand clapping. They consider a conflict between average noise intensity and synchronization. They discuss a mechanism of hand clapping period doubling by individuals that helps the group achieve synchronization. Their results offer a novel route to synchronization, not observed in physics or biological systems by the time of publication.

Synchronization only happens in self-sustained systems [21]. The authors describe self-sustained oscillators mathematically as an autonomous (i.e. without explicit time dependence) nonlinear dynamical systems. Phase of an oscillator is con-

\footnotetext{
${ }^{2}$ Christian Huygens is probably the first scientist who observed and described the synchronization phenomenon.
} 
sidered neutrally stable. This means that a slight perturbation can change the phase while the amplitude is stable and is not affected by external perturbations. This property provides the oscillator the ability to synchronize [21].

Synchronization of two metronomes is investigated in [22]. The authors address the problem of analytical study of in-phase synchronization for the model of two metronomes an the common support proposed in [23]. The authors proved the existence of an in-phase regime and they proved that for when the angle difference of metronomes was zero $\left(\phi_{1}-\phi_{2}=0\right)$, the sum of angles $\left(\phi_{1}+\phi_{2}\right)$ has a periodic regime.

The synchronization of an array of clocks hanging from an elastically fixed horizontal beam is studied in [24]. The beam is considered as a rigid body connected to a spring and a damper. Different types of synchronization are observed in that research: Symmetrical Synchronization, Complete Synchronization and De-synchronous Behavior. Synchronization of two and more metronomes is considered in [23]. Synchronization of coupled mechanical metronomes is also studied in [25]. The authors study synchronization by means of numerical simulations showing the onset of synchronization for two, three and 100 globally coupled metronomes.

Crowd synchrony on London Millennium Bridge [26] is investigated in [27]. Sufficient conditions for controlled synchronization of non-linear systems is provided in [28]. An attempt to provide a general formalism for synchronization in dynamical systems is shown in [29]. Frequency and coordinate synchronizations are considered in that article. It is mentioned that synchronization as a phenomenon should be considered in context and depends on the view. A system showing synchronization viewed from a view point, might not seem having synchronization from another point of view.

A sample of synchronization is observed in various species. For instance, banded mongoose groups show high degree of birth synchrony to avoid the negative effects 
of competition with other females [30]. Synchronization of time-delayed systems is discussed in [31]. A brief introduction to the theory of synchronization of selfsustained oscillators is presented in [32].

Synchronization is investigated in other fields such as Small-World networks [33-35], in weighted complex networks [36-38] and in dynamical networks [39]. Phase synchronization of weakly coupled self-sustained chaotic oscillators is investigated in [40]. The exact mechanisms of generation of epileptic seizures in human brains is still uncertain. Nevertheless, it is widely accepted that an abnormal synchronization of firing neurons causes epileptic seizures. To investigate this problem, phase synchronization between different regions of the brain is measured in many researches $[41,42]^{3}$.

\section{Chaos}

Dissipative systems with a nonlinear time-delayed feedback or memory can produce chaotic dynamics $[43,44]$. The effect of the delay on the dimension of these chaotic attractors is shown in [45]. Delay systems generically have families of periodic solutions, which are reappearing for infinitely many delay times. As delay increases, the solution families overlap leading to increasing coexistence of multiple stable as well as unstable solutions [46]. Anticipating chaotic synchronization is discussed in [47].

\section{E. Self-Adjusting Systems and the Edge of Chaos}

Packard [48] showed adaptation to the edge of chaos in cellular automata rules with genetic algorithms. Some of his results were later disputed in [49]. Co-evolution to the edge of chaos is discussed in [50]. Edge of chaos has been found to be the

\footnotetext{
${ }^{3}$ This seems to be a potential line of research. If controlling this synchronization can be under control or be interfered by some means, there might be a tool for controlling epileptic seizures.
} 
optimal setting for control of a system [51]. A self-adjusting system is a system in which the control of a parameter value depends on previous states of the system [52]. The authors in [53] describe adaptation to the edge of chaos in logistic map. They believe that adaptation to the edge of chaos is a generic property of the systems with a low-pass filtered feedback. They believe that this property is independent of the form of the feedback and the system under study. The findings have also been confirmed experimentally with Chua's circuit [54]. In [55] conserved quantities are used for investigating adaptation to the edge of chaos. The phrase Edge of Chaos was originally proposed by Chris Langton in 1990 in the area of cellular automata [56] although others mentioned the same at the same time [57]. Guiding an adaptive system through chaos is also considered in [58]. The topic is also discussed in [59-62].

\section{F. Purpose of this Research}

We would like to consider oscillators and the effect when they are coupled through a medium. We would study the synchronization phenomenon between oscillators and we would like to study mechanical oscillators in an experimental settings and we consider the important factors affecting the synchronization. We also study the case in which we interfere with the coupling. For this case, we use the concept of forced oscillators and use mechanical metronomes located on a moving base. We study the effects of movements of the base on the behaviors of oscillators. This makes the whole set a rich dynamical system that has many potentials to be explored. We will consider the case where the system can adjust itself. We study how the system evolves in time when some parameters of the system are adjusted by states of the system itself. 


\section{CHAPTER II}

\section{EXPERIMENTAL SETTINGS}

We used an experimental setting for this research. The description and details of these settings are provided in this chapter.

\section{A. Description}

We use mechanical wind-up metronomes as oscillators that are mechanically coupled through a moving base. The metronomes are Wittner's Super-Mini-Taktell (Series 880) and are claimed to be the world's smallest pendulum metronomes. Another part of the system is in charge of reading the angle values of metronome rods in time. A schematic of the system is shown in Fig. 1 and some images of the experimental settings are shown in Fig. 2.

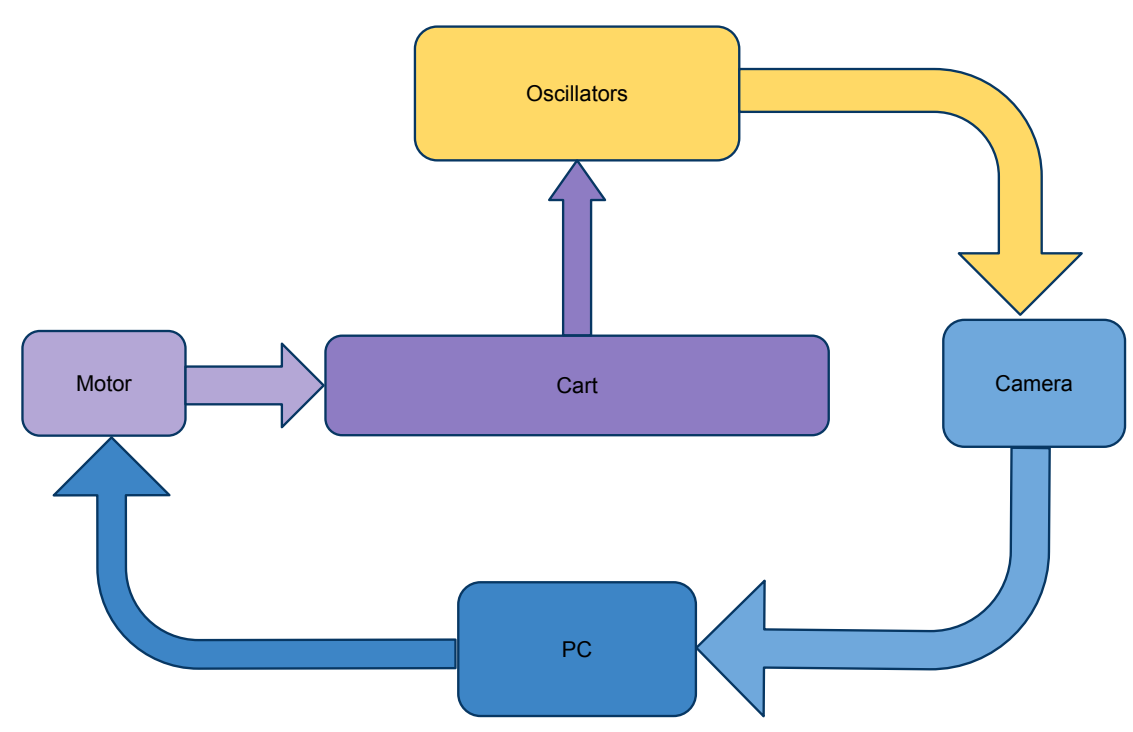

Fig. 1. The schematic of the system 

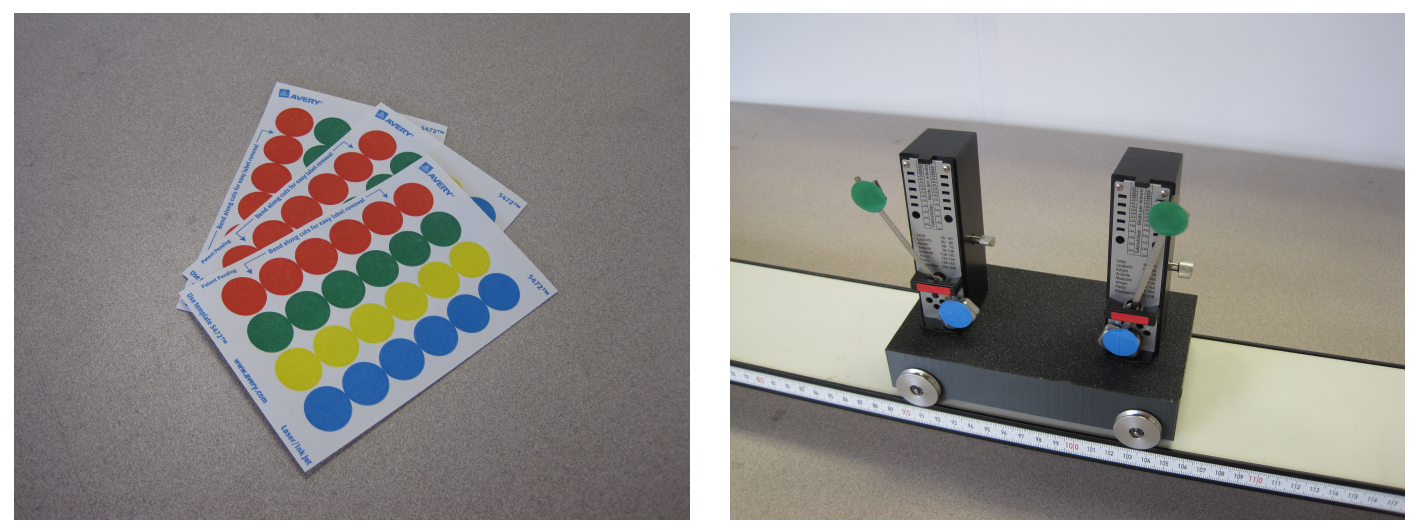

Stickers used for detection of points in Two metronomes on a freely moving image processing passive cart
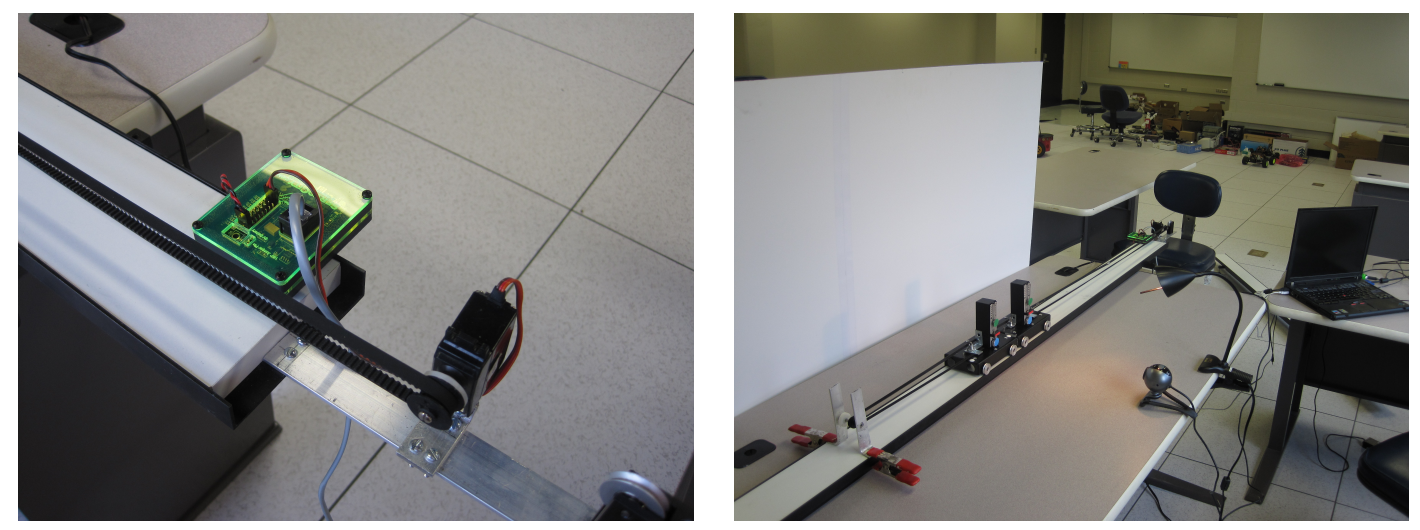

Servo motor and the controller for mov- A general view of the whole settings ining the active cart cluding the camera, the track, the carts, the motor and the PC

Fig. 2. Experimental settings

\section{B. Settings}

One of the main factors we had in mind was that to build a robust and reliable setting that is easily available and possible to use. We needed to read the values of angles at different times and one of the first candidates was using encoders for reading the values. As the encoders might have affected the mechanics of the metronomes and hence might have affected the dynamics, we preferred to choose an approach for reading the values of angles that does not affect the dynamics of the system. 
We selected a vision and an image processing approach so that we track the rod; for instance, the conservation of mechanical energy using video analysis has been investigated in [63]. We used simple colored stickers so that the vision system can use the color for tracking the position. The stickers are circles for which the radius is 9.4 $\mathrm{mm}$ and the choice of colors are red, green, yellow and blue. Regarding the position of the stickers, the center of each sticker was marked and a tiny hole was made in the center. This hole was used to align the position of the sticker on the center of the rod.

\section{Image Processing}

The main image processing library that is used in this system is OpenCV ${ }^{1}$. A simple USB webcam is used to capture the image and the capture frame rate is around 20 to 40 frames per second ${ }^{2}$. We do not use a pre-recorded movie for processing so that we can have real-time control on the system. The image processing module has these main sections

1. Capture Image: The program captures the image from the webcam

2. Track Colored Stickers: The markers are tracked

3. Find Values: The values, such as angles of metronomes, are calculated based on the detected values for positions of the markers

\footnotetext{
${ }^{1}$ OpenCV is a computer vision library originally developed by Intel. It is free for use under the open source BSD license. The library is crossplatform and it focuses mainly on real-time image processing. It is available at http://opencv.willowgarage.com/

${ }^{2}$ This is a rough estimate and is extracted based on experiments in normal conditions. It is changed according to the settings of the program and the specifications of the camera used for the image processing.
} 
4. Evaluate Values: The values, such as angles of metronomes, are evaluated and the unaccepted data is discarded

5. Store Values: The values are stored in the memory

6. Apply Control: Control commands are issued (such as the commands for the moving cart)

7. Save Values: The values are saved on the data storage

In order to track the objects in real-time, an algorithm is in charge of looking for proper pixels in a certain window around the object that is being tracked. With enhanced settings, we used more than one color sticker for each metronome rod so that we increase the accuracy of angle detection (color image processing has been used in some applications such as [64]). The pseudo-code used for the image processing module is shown in Algorithm 1. In this pseudo-code we have described the basic steps required for the image processing module. A sample of the perceived image is shown in Fig. 3.

To present the range of values detected from the vision, the values for a short period of time are shown in Fig. 4. This picture provides the rough idea of the range of numbers used for deducing the angles of the rods. A calibration is done to deduce the distances in mm based on the detected pixels. For this case every 41 pixels correspond to $18.8 \mathrm{~mm}$.

In another experiment the frequency of the metronome was reduced to $0.4 \mathrm{~Hz}$. The values and a short period of time are shown in Fig. 5. For this case every 66 pixels correspond to $18.8 \mathrm{~mm}$. 


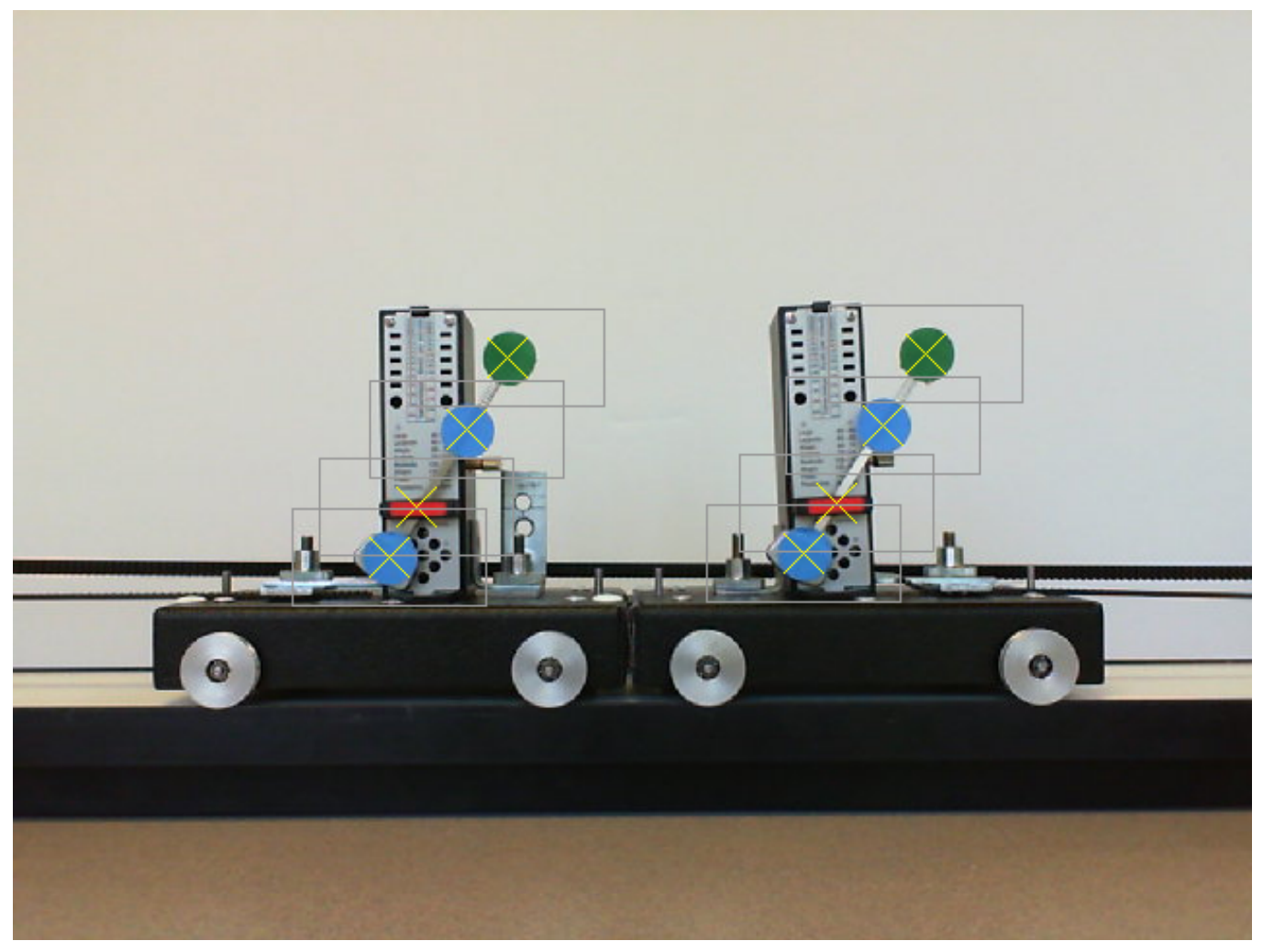

Fig. 3. Two metronomes on two connected carts. The yellow crosses show the center of mass of the detected stickers. Also there are gray rectangles around each of the stickers which show the area to be searched at each step. In this setting we have used color stickers to detect the position of the carts as well.

\section{Finding the Angle}

When using more than two stickers on the rod, we need to find the least squares regression line. We use the following formula for finding the angle of the metronome $\operatorname{rod}$

$$
\theta=\tan ^{-1}\left(\frac{n \sum_{i=1}^{n} x_{i} y_{i}-\sum_{i=1}^{n} x_{i} \sum_{i=1}^{n} y_{i}}{n \sum_{i=1}^{n} x_{i}{ }^{2}-\left(\sum_{i=1}^{n} x_{i}\right)^{2}}\right)
$$

in which $n$ is the number of stickers and $x_{i}$ and $y_{i}$ are the $x$ and $y$ of the $i$ th sticker 


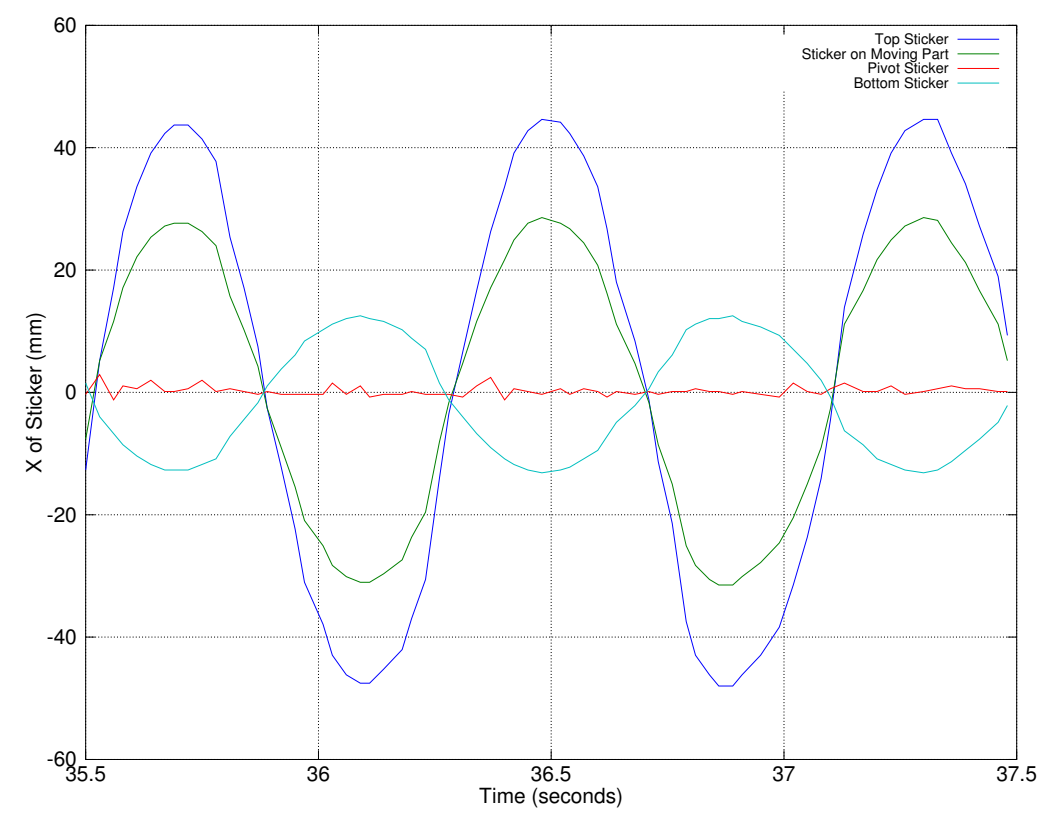

Fig. 4. A short time frame of the values from investigation of a metronome for different stickers. Four stickers are used on a metronome: one blue color is used on the very top of the rod, one green color is on the moving weight and one blue color on the bottom of the rod. One red sticker is also used on the pivot of the rod. All the values are extracted from the image and converted to $\mathrm{mm}$ based on calibrations. The metronome is located on a solid base and the frequency is 1 $\mathrm{Hz}$ (which corresponds to 120 beats per minute).

in pixels.

\section{Image Processing Calibration}

A square is used for which the length of each side is $72.4 \mathrm{~mm}$. We marked each corner with a sticker and added a sticker to the geometrical middle of the square. The settings and the data are shown in Fig. 6. 


\section{Validating the Results}

The image processing module might occasionally lose track of stickers. To prevent the consequences of this problem and to validate the results, correlation of the positions of the detected stickers are calculated as below

$$
r_{x y}=\frac{n \sum x_{i} y_{i}-\sum x_{i} \sum y_{i}}{\sqrt{n \sum x_{i}^{2}-\left(\sum x_{i}\right)^{2}} \sqrt{n \sum y_{i}^{2}-\left(\sum y_{i}\right)^{2}}}
$$

in which $n$ is the number of stickers and $x_{i}$ and $y_{i}$ are the $x$ and $y$ of the $i$ th sticker in pixels. If correlation is more than a certain threshold, the sample is accepted, used for updating the values and stored. If the correlation is smaller than the threshold, then there might be one possibility: The positions of the detected stickers are too close to a vertical line (which reduces the correlation). In that case, the detected points are virtually rotated $\frac{\pi}{4}$ radians and the correlation is calculated again. If the correlation is more than the threshold, it means that the detected points have been sufficiently close to a line and the line has been vertical on the first round. If correlation is less than the threshold even in the second round, then it shows error in detecting the stickers and the sample is discarded. 


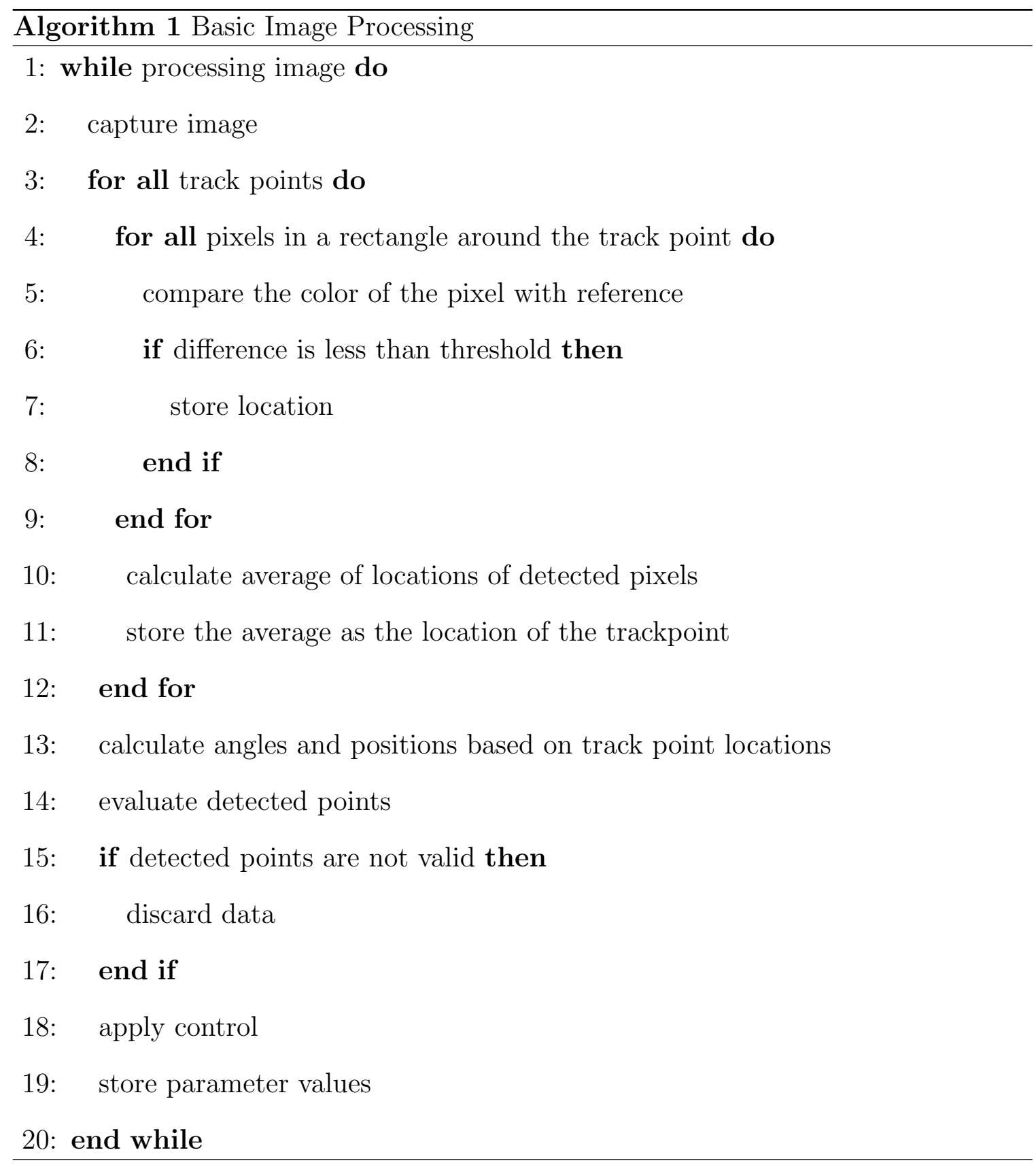



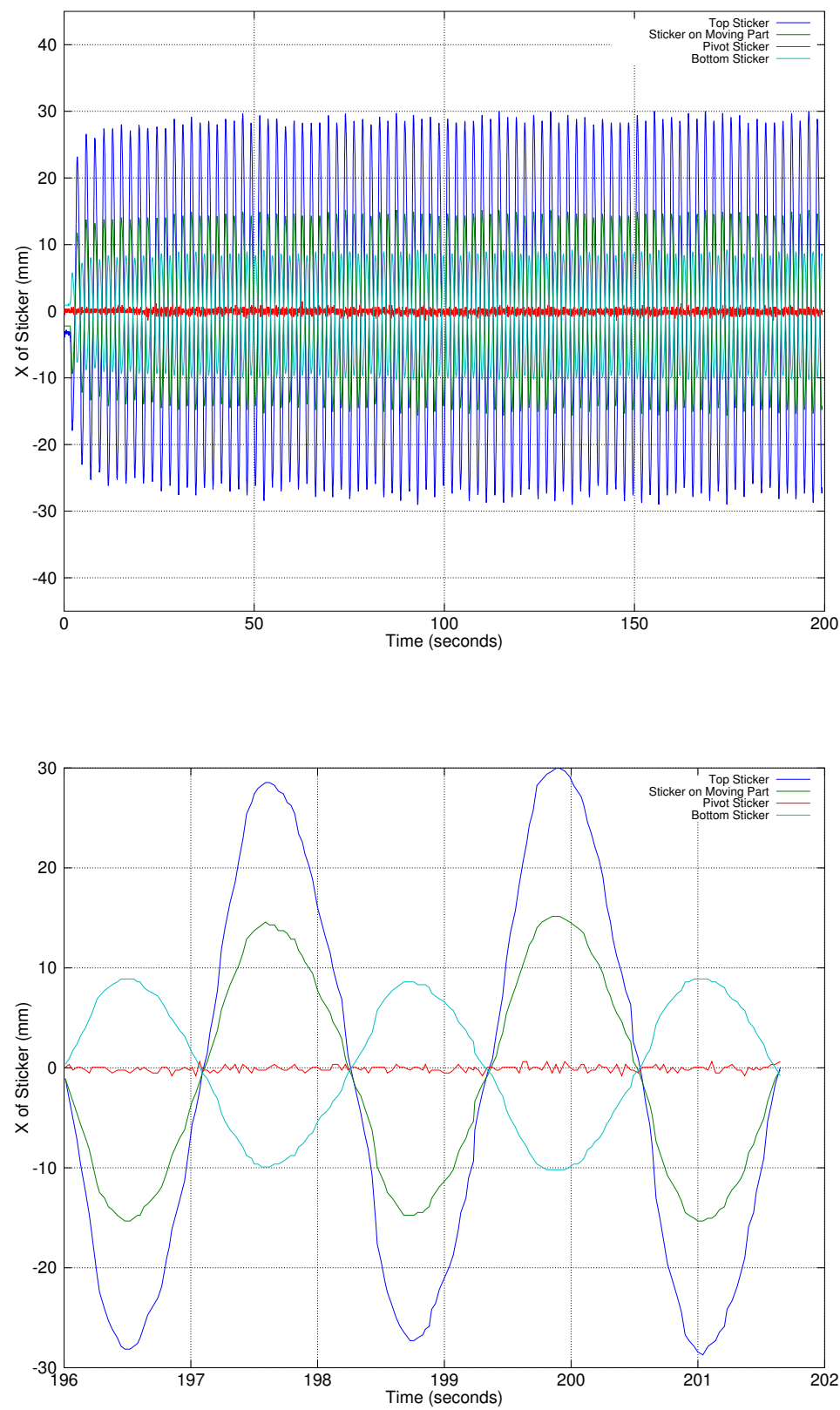

Fig. 5. The values from investigation of a metronome to show the positions of the stickers. Four stickers are used on a metronome: one green color sticker is on the moving weight, one blue color is used on middle of the rod and one blue color on the bottom of the rod. One red sticker is also used on the pivot of the rod. All the values are read from the image and are converted to $\mathrm{mm}$. The metronome is located on a solid base and the frequency is $0.4 \mathrm{~Hz}$ (which corresponds to 48 beats per minute). 

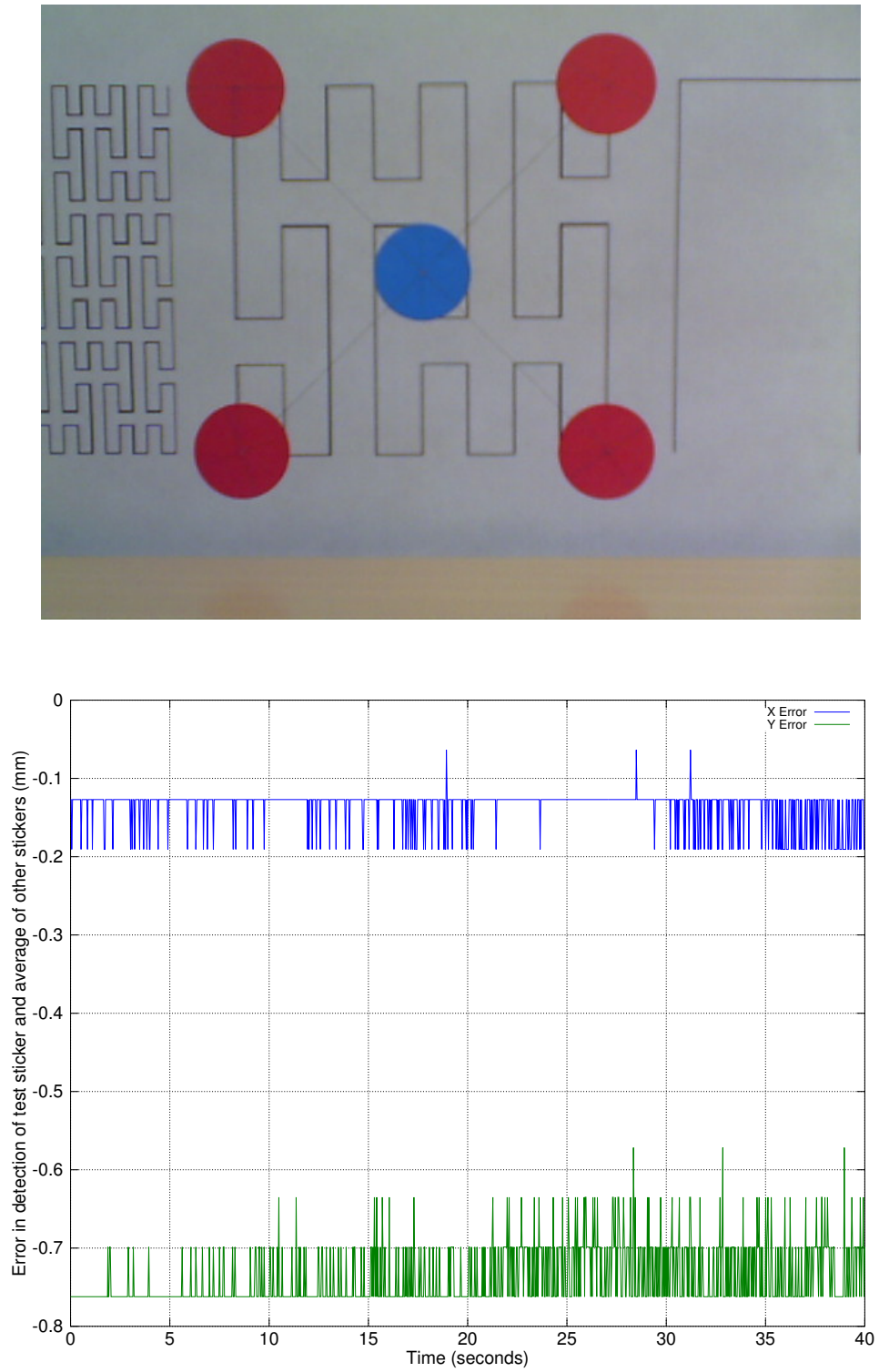

Fig. 6. The settings and the errors for calibration test. In this test we have four red stickers on the corners of a square and one blue sticker in the middle of the square. The length of each side of the square is $72.4 \mathrm{~mm}$. For this test every 74 pixels correspond to $18.8 \mathrm{~mm}$. 


\section{Active Cart}

To determine the speed of the cart when moved by the servo motor, a simple test is done in which the cart moves to the right and left with the fastest possible speed. The position of the cart vs. time is shown in Fig. 7.

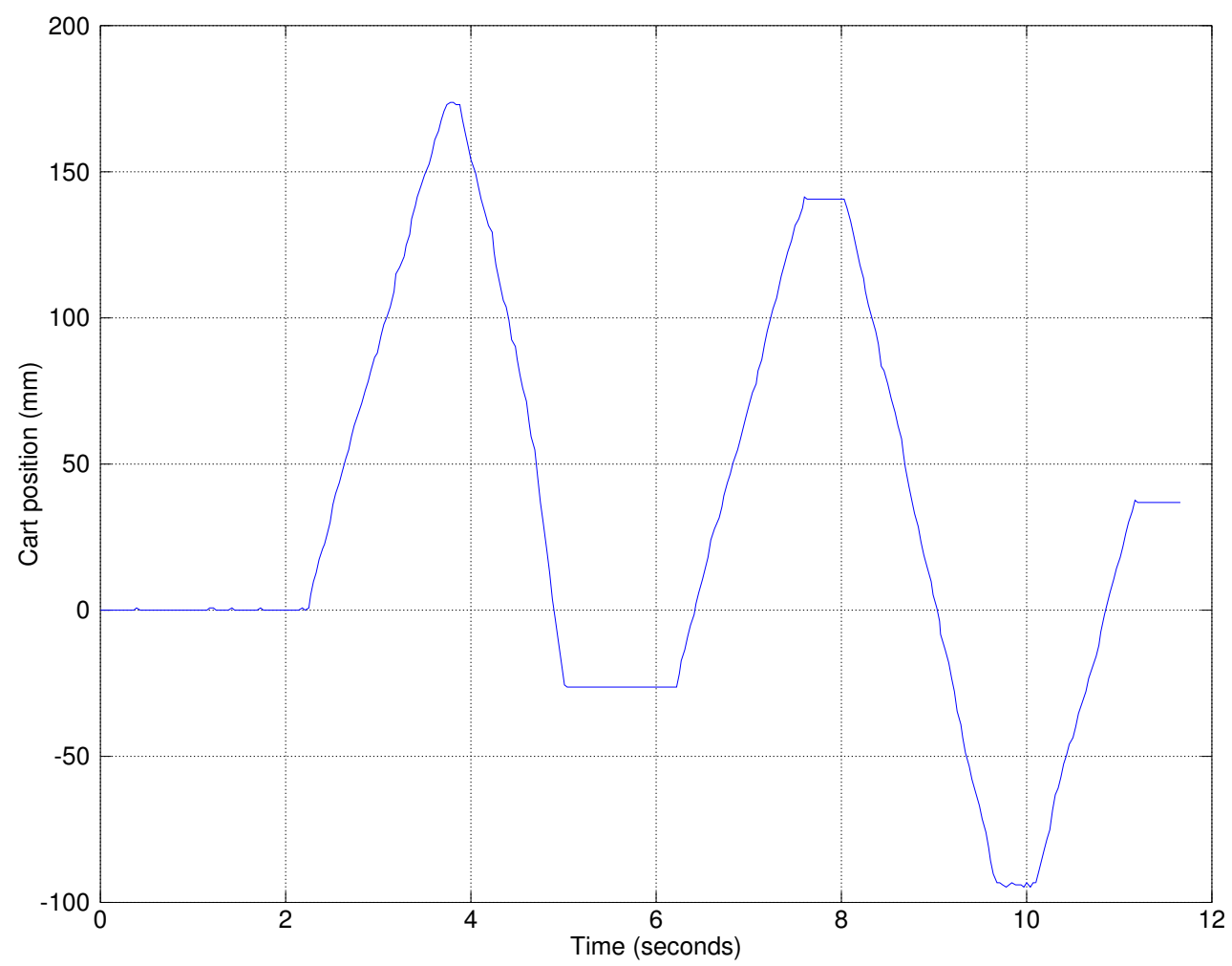

Fig. 7. The position of the cart in a test. The cart was moved with the fastest possible speeds to right and left. 


\section{E. Experimental Results}

For one of the settings, a simple metronome is placed over a solid base. The phase of the metronome is shown in Fig. 8 and a partial view of the time series is shown in Fig. 9.

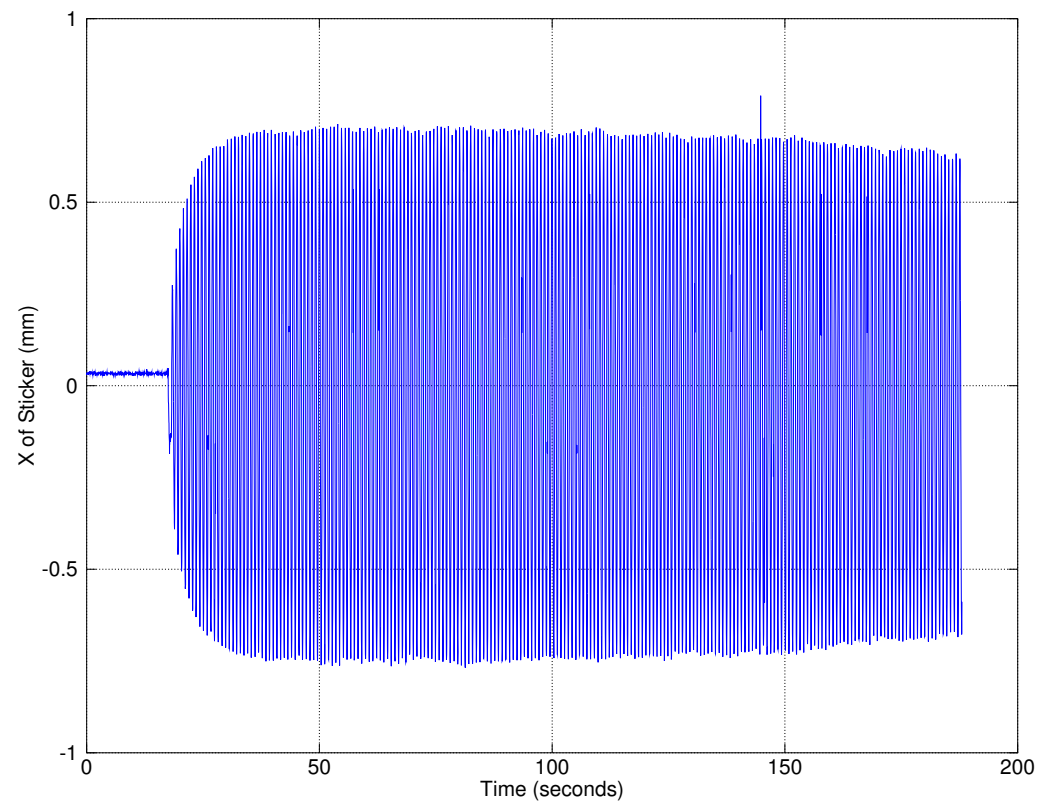

Fig. 8. The phase of a metronome in time showing the transient region. The transient region at the beginning shows the beginning of the experiment in which the rod is started from the rest position. The phase is later absorbed to the limit cycle (the stable region after the transient). The angle is compared to the vertical line. Hence positive values of angle show that the rod is bend towards right and negative values show bending towards left. Zero value shows that the rod is vertical. The metronome is located on a solid base and the frequency is 1 $\mathrm{Hz}$ (which corresponds to 120 beats per minute). 


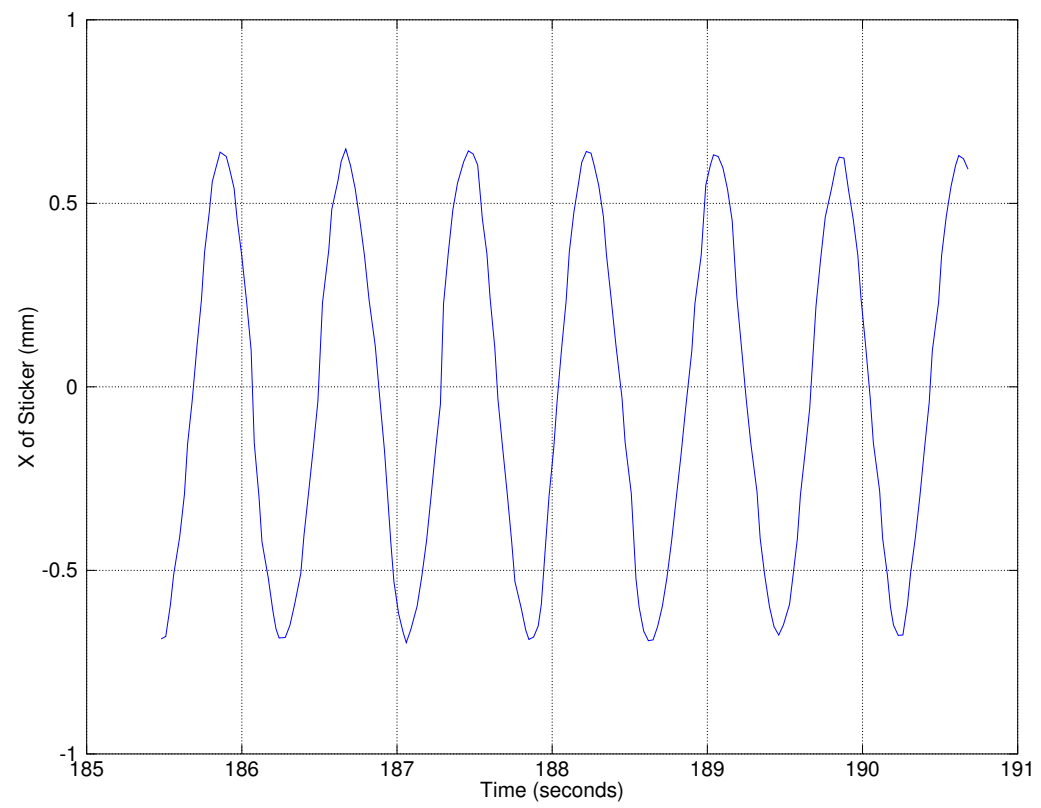

Fig. 9. A short time frame of the metronome phase showing that the shape of the wave is not completely a sine wave. This agrees with the model and simulations (the shape of the wave depends on the parameters of the system such as escapement of the metronome). The average capture frame rate is about 32 frames per second. The metronome is located on a solid base and the frequency is $1 \mathrm{~Hz}$ (which corresponds to 120 beats per minute). 
For another setting we used a much lower frequency for the metronome. In this case also a simple metronome is placed over a solid base. The phase of the metronome is shown in Fig. 10 and a partial view of the time series is shown in Fig. 11.

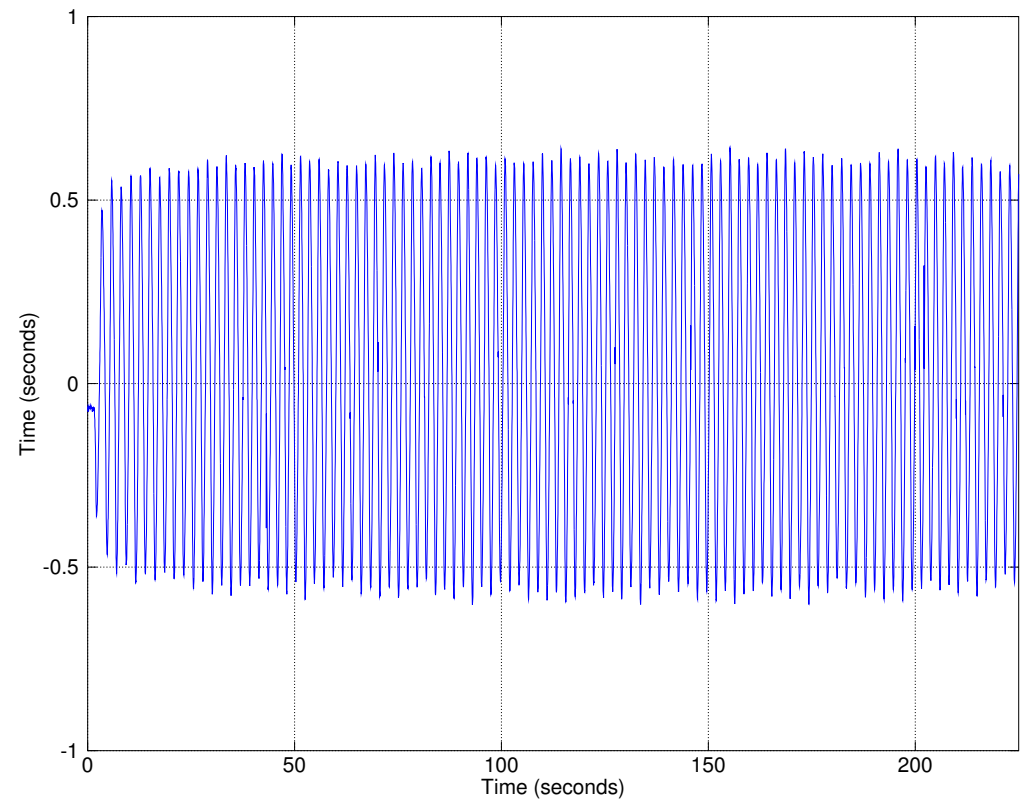

Fig. 10. The phase of a metronome in time when the metronome is located on a solid base. The angle is compared to the vertical line. Hence positive values of angle show that the rod is bend towards right and negative values show bending towards left. Zero value shows that the rod is vertical. The metronome is located on a solid base and the frequency is $0.4 \mathrm{~Hz}$ (which corresponds to 48 beats per minute). 


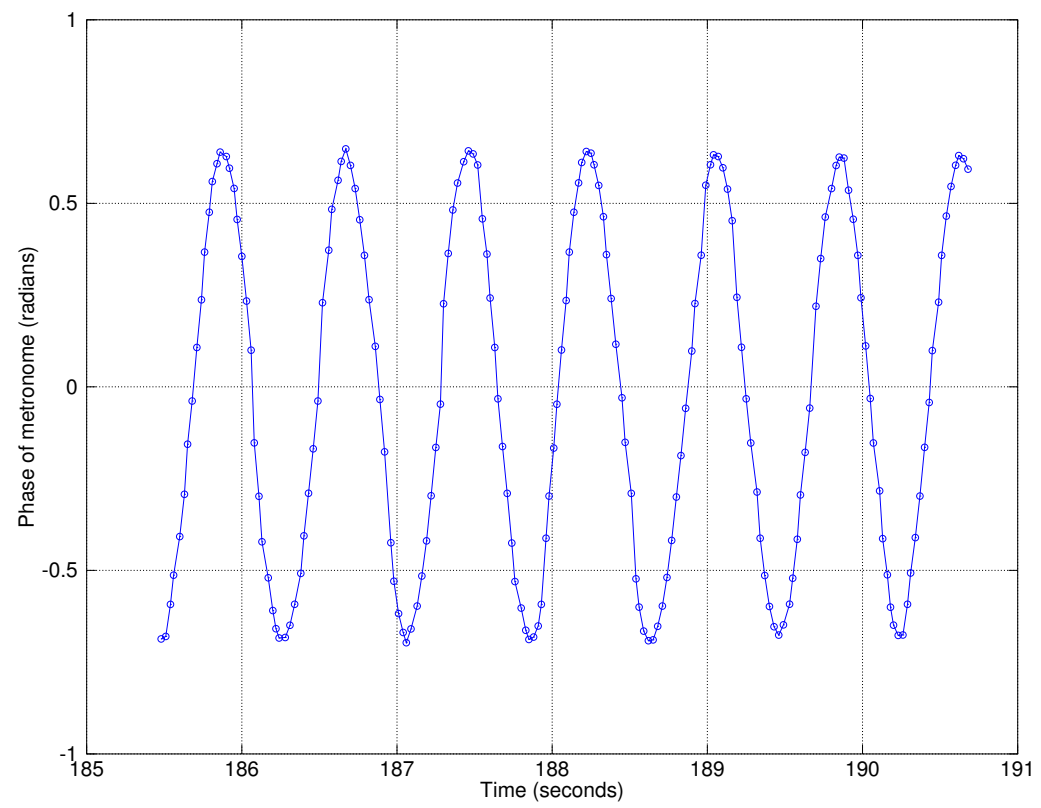

Fig. 11. A short time frame of the metronome phase showing sample points. The shape of the wave is not completely a sine wave and this agrees with the model and simulations (the shape of the wave depends on the parameters of the system such as escapement of the metronome). The average capture frame rate is about 32 frames per second. The metronome is located on a solid base and the frequency is $0.4 \mathrm{~Hz}$ (which corresponds to 48 beats per minute). 


\section{Storing the Images}

In order to verify the results, we set up experiments in which we stored the images while processing them. This would help to find potential flaws of the image processing module. The drawback of this approach is that it imposes overhead on the system and decreases the accuracy because of less processing speed. A time frame sample of the signal is shown in Fig. 12.

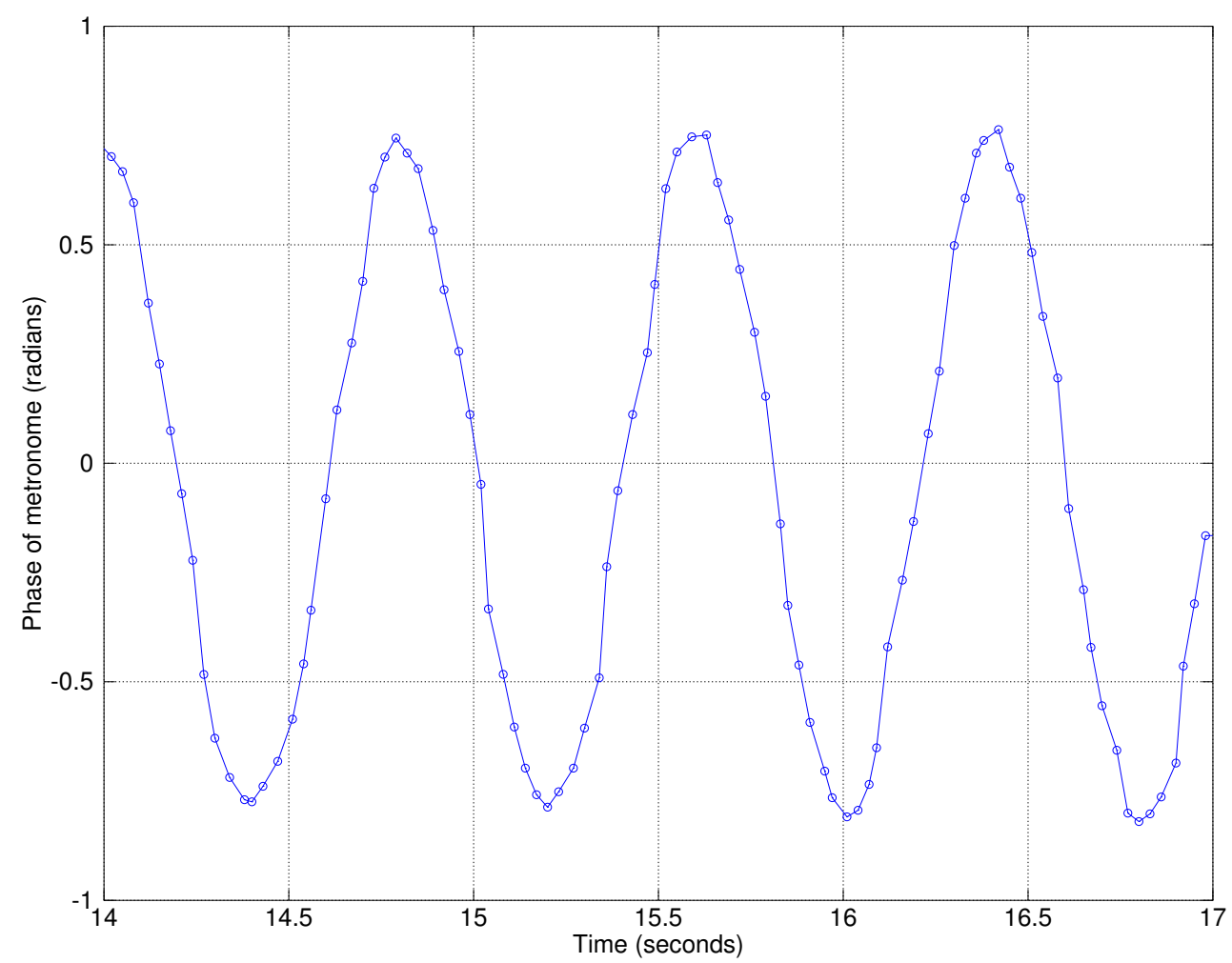

Fig. 12. A short time frame of the metronome phase to show more details with sample points. In this experiment the images are stored in the memory for debugging purposes. The metronome is located on a solid base and the frequency is 1 $\mathrm{Hz}$ (which corresponds to 120 beats per minute). 


\section{Pivot Point}

In Fig. 13 the distance of the detected pivot point from the regression line is presented.
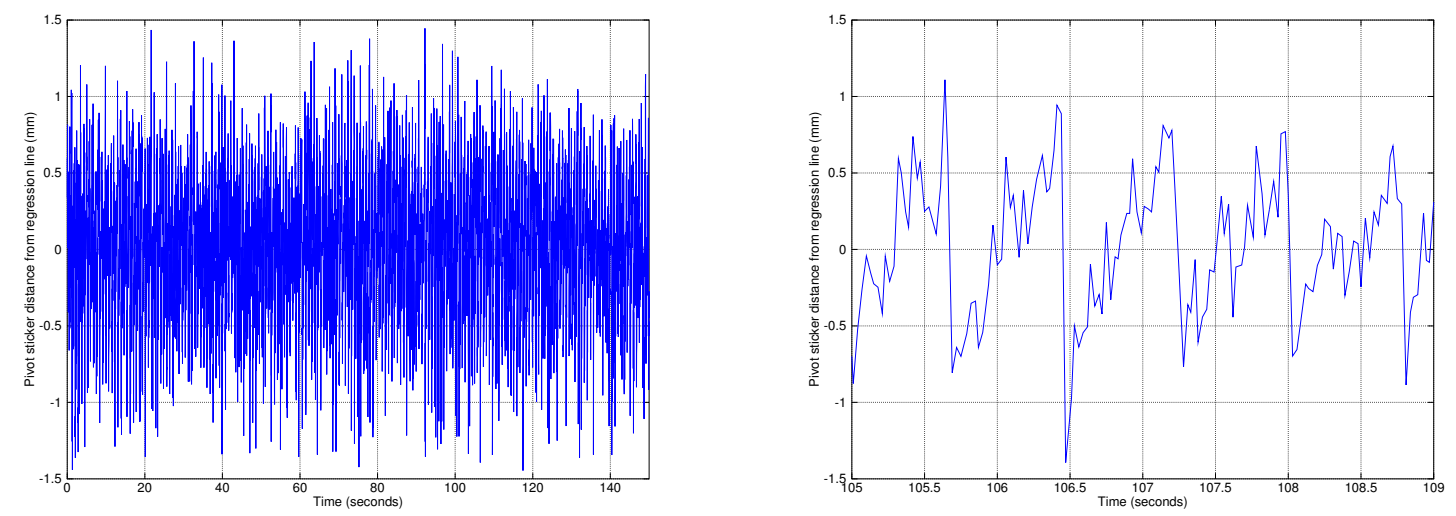

Fig. 13. The distance of the detected pivot point from the regression line of all the stickers for higher frequency. A short time frame is shown on the right. The metronome is located on a solid base and the frequency is $1 \mathrm{~Hz}$ (which corresponds to 120 beats per minute).

The same test was done with a lower frequency of the metronome. In Fig. 14 the distance of the detected pivot point from the regression line for the whole test is presented. 

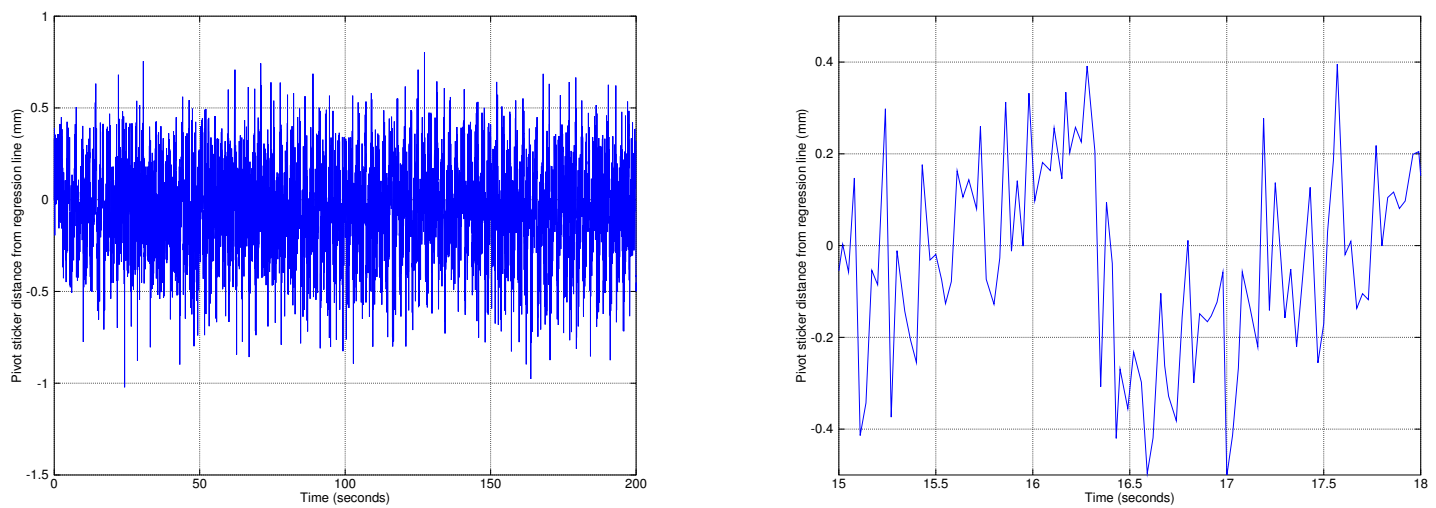

Fig. 14. The distance of the detected pivot point from the regression line of all the stickers for lower frequency. The distance of the detected pivot point from the regression line of all the stickers for lower frequency. A short time frame the right. The metronome is located on a solid base and the frequency is 0.4 $\mathrm{Hz}$ (which corresponds to 48 beats per minute). 


\section{Using Hue-Saturation-Value for Tracking Colors}

We use Hue-Saturation-Value for tracking the colors in image processing. For this case we convert the RGB (Red-Green-Blue) of the original pixel to HSV (Hue-SaturationValue). Then we compare the HSV of the search pixels of the image frames with the original pixels. If $\mathrm{H}, \mathrm{S}$ and $\mathrm{V}$ are in a certain range of difference, we consider the detected pixel as a part of the sticker. The ranges of parameter for thresholds are determined by the experiment. The algorithm for converting RGB to HSV is shown in Algorithm 2.

\section{F. Base Setups}

Some properties of the base, such as damping, play an important role on synchronization $[23,65]$. Hence, we have tried various base settings for our experiments. In a passive base, the base can move almost freely. We tried to reduce the friction as much as possible to avoid possible interferences. In another setting for the base, it can move according to commands issued from the controller program. When running tests, we set the metronomes' rods to random initial angles and then release them.

\section{G. Conclusions}

In this chapter we described experimental settings that were used in this project. We described the image processing module and how we use the vision to gain sensory information. We described the details of the implementation and some important factors, such as how to find the angles of mechanical oscillators and how to validate the results. The one degree of freedom cart was introduced and the experimental results of running the set were provided. 


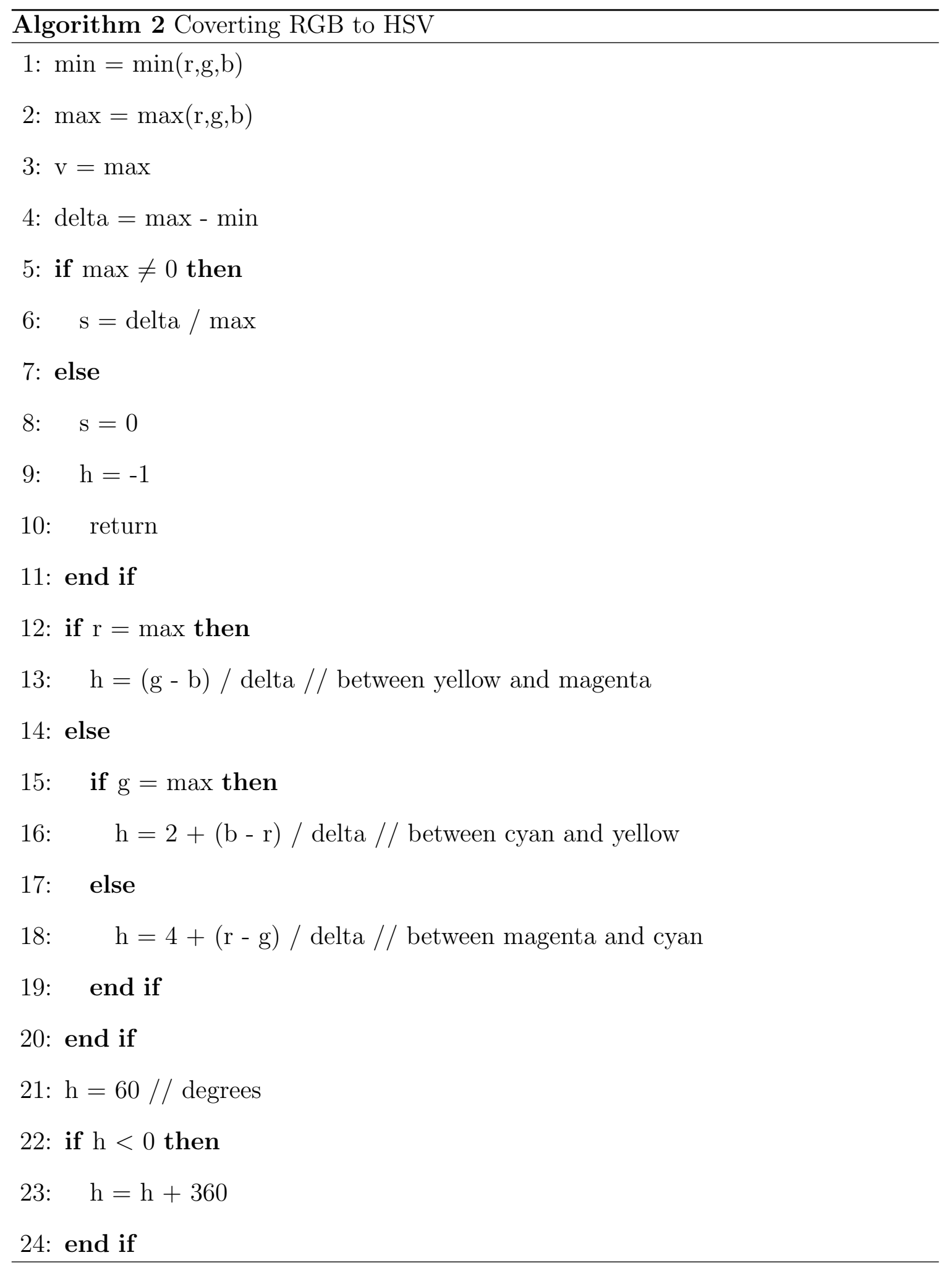




\section{CHAPTER III}

\section{SYNCHRONIZATION}

Different settings and sets of parameters were used to study synchronization. Some of them and the results are described in this chapter.

\section{A. Model of Oscillators}

The equation that governs the motion of a single metronome is described as below $[23,25]$

$$
\frac{d^{2} \theta}{d t^{2}}+\underbrace{\frac{m r_{c . m .} g}{I}}_{k} \sin (\theta)+\epsilon\left[\left(\frac{\theta}{\theta_{0}}\right)^{2}-1\right] \frac{d \theta}{d t}=0
$$

in which $\theta$ is the phase of the metronome (angle made with the vertical line), $I$ is the moment of inertia of the pendulum, $m$ is the mass of the pendulum, $r_{c . m}$. is the distance of the pendulum's center of mass from the pivot point, $\epsilon$ specifies the effect of escapement and damping, $\theta_{0}$ is the van der Pol term and $g$ is the acceleration of the

gravity. $k=\frac{m r_{c . m . g}}{I}$ determines the frequency of oscillation. The damping function $D(\theta)$ is defined as $[25]$

$$
D(\theta)=\left(\frac{\theta}{\theta_{0}}\right)^{2}-1
$$

and with small $\epsilon$, this term produces small oscillations with an amplitude of approximately $2 \theta_{0}$ in an isolated oscillator [23]. 


$$
\begin{aligned}
& \frac{d \theta}{d t}=\gamma \\
& \frac{d \gamma}{d t}=-\underbrace{\left(\frac{m r_{c . m . g}}{I}\right)}_{k} \sin (\theta)-\epsilon\left[\left(\frac{\theta}{\theta_{0}}\right)^{2}-1\right] \gamma
\end{aligned}
$$

in which $\gamma$ is the rotational speed and $\epsilon$ specifies the effect of escapement and damping. When $\epsilon=0$, we do not have a limit cycle and we have infinite cycles as shown in Fig. 15. In this case the phase is a sine wave. When $\epsilon \neq 0$, we have a limit cycle as shown in Fig. 16. In this case the phase is not a sine wave and changes the shape depending on the value of $\epsilon$.
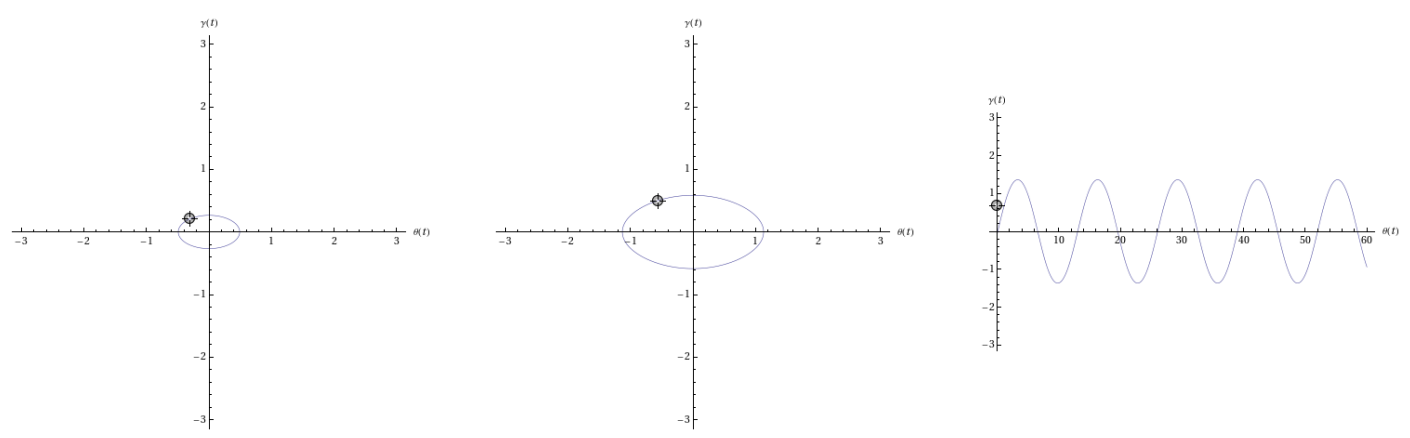

Fig. 15. The trajectory of the phase when the base does not move with $\epsilon=0$. In this case $k=0.3, \epsilon=0$ and $\theta_{0}=\frac{\pi}{4}$ 

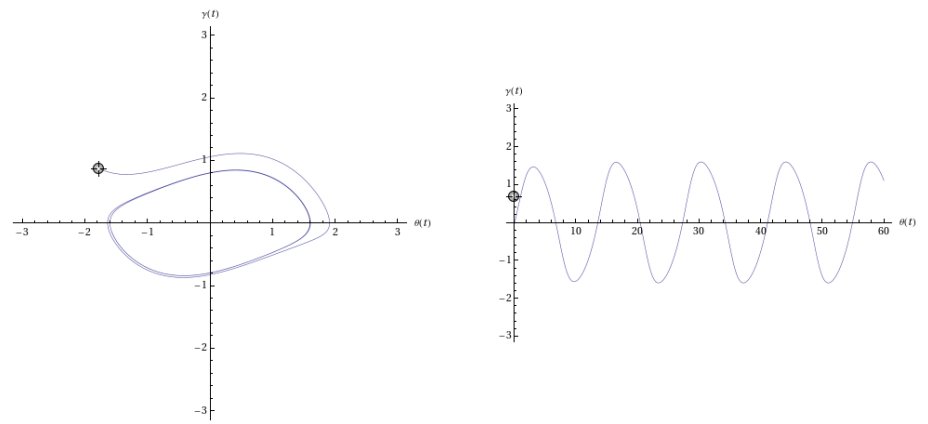

Fig. 16. The phase when the base does not move with $\epsilon \neq 0$. In this case $k=0.3$, $\epsilon=0.2$ and $\theta_{0}=\frac{\pi}{4}$ 
B. Oscillators on a Passive Base

When the oscillators are located on a passive base ${ }^{1}$, they have the possibility of synchronization. A schematic of the settings is shown in Fig. 17.

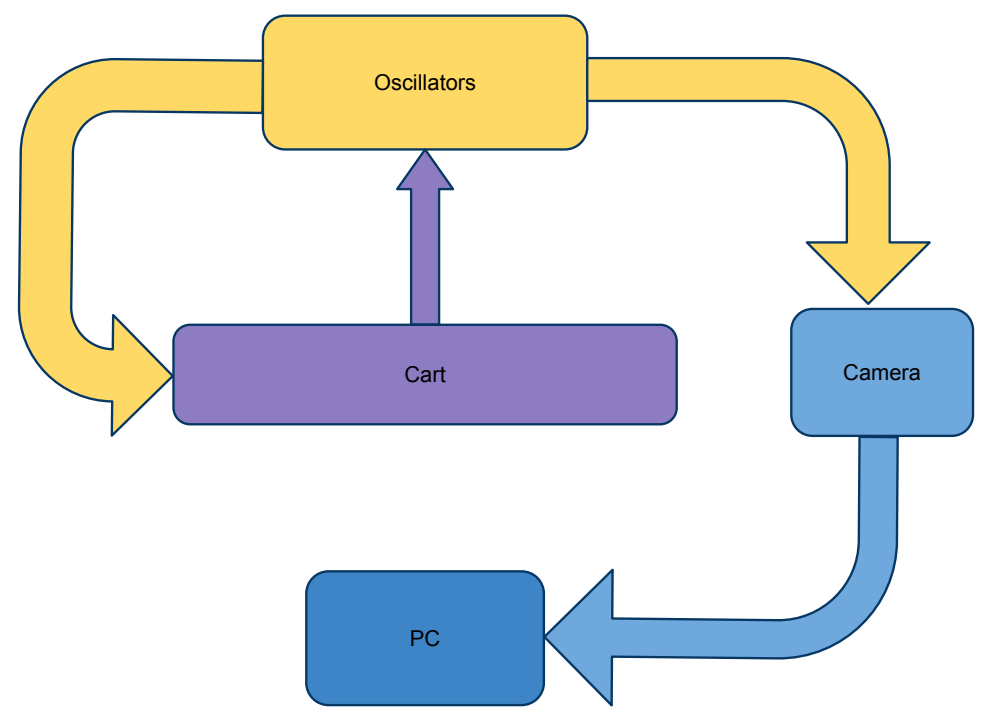

Fig. 17. The schematic of the system for two metronomes located on a passive base. The metronomes move the base and the movements of the base affect the metronomes. The camera and the $\mathrm{PC}$ are only for observation.

The equations of motion in this case are as follows [23]

${ }^{1}$ By a passive base we mean a common base on which the metronomes are located and moves freely. A sample is shown in Fig. 2. 


$$
\begin{aligned}
& \frac{d^{2} \theta_{1}}{d \tau^{2}}+(1+\Delta) \sin \left(\theta_{1}\right)+\mu\left[\left(\frac{\theta_{1}}{\theta_{0}}\right)^{2}-1\right] \frac{d \theta_{1}}{d \tau}-\beta \cos \left(\theta_{1}\right) \frac{d^{2}}{d \tau^{2}}\left(\sin \left(\theta_{1}\right)+\sin \left(\theta_{2}\right)\right)=0 \\
& \frac{d^{2} \theta_{2}}{d \tau^{2}}+(1-\Delta) \sin \left(\theta_{2}\right)+\mu\left[\left(\frac{\theta_{2}}{\theta_{0}}\right)^{2}-1\right] \frac{d \theta_{2}}{d \tau}-\beta \cos \left(\theta_{2}\right) \frac{d^{2}}{d \tau^{2}}\left(\sin \left(\theta_{1}\right)+\sin \left(\theta_{2}\right)\right)=0
\end{aligned}
$$

in which $\tau=\omega t$ is a dimensionless time variable, $\omega^{2}=\frac{m r_{c . m . g}}{I}$ is the square of the average angular frequency of the uncoupled, $\Delta \approx \frac{\omega_{1}-\omega_{2}}{\omega}$ is the relative frequency difference between the oscillators, small amplitude oscillator without damping or driving, $\theta_{0}$ is the van der Pol term and $\beta$ is the coupling parameter

$$
\beta=\left(\frac{m r_{c . m .}}{M+2 m}\right)\left(\frac{r_{c . m .} m}{I}\right)
$$

in which $M$ is the mass of the base, $I$ is the moment of inertia of the pendulum, $m$ is the mass of the pendulum (masses of the pendulums is considered to be the same), $r_{c . m}$. is the distance of the pendulum's center of mass from the pivot point. For low damping of the coupling medium we may observe in-phase synchronization and when the damping of the coupling medium is high, we can observe anti-phase synchronization. The simulations with various values of $\beta$ and the phase transition are shown in Fig. 18 (the plots are shown for times when the transient is relaxed). 

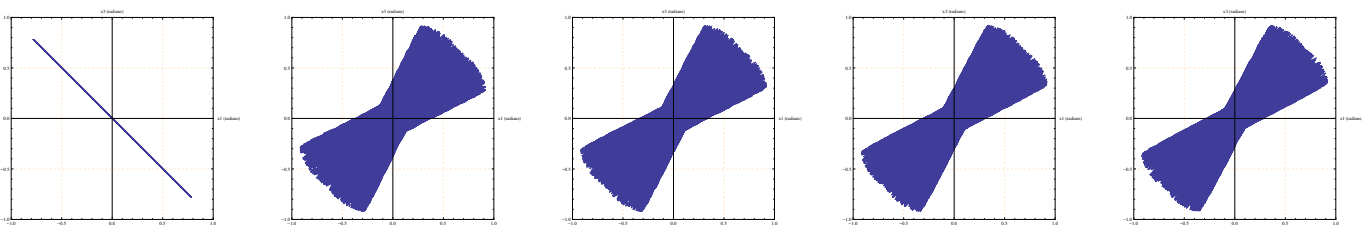

0.056

0.057

0.058

0.059

0.060
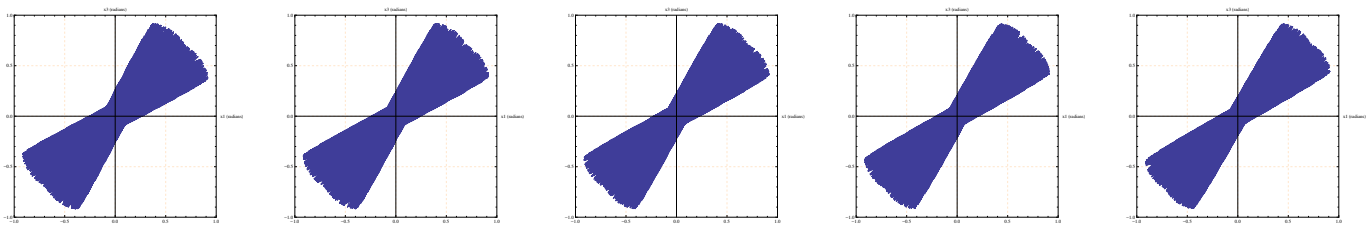

0.061

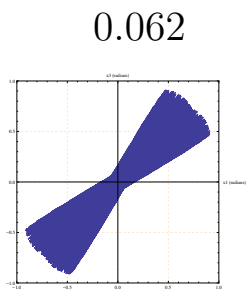

0.063

0.064

0.065
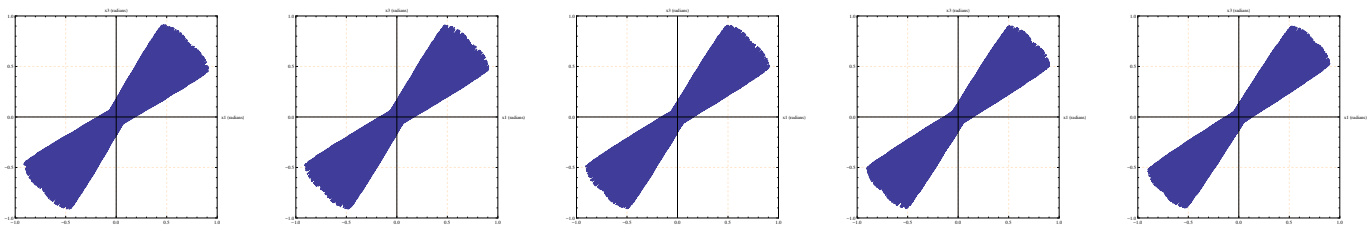

0.066

0.067

0.068

0.069

0.070
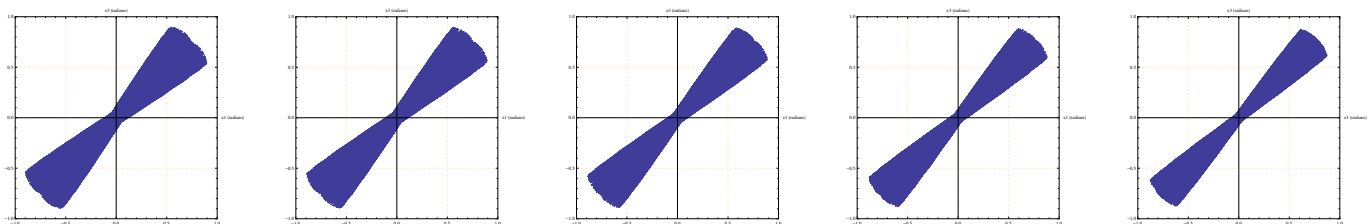

0.071

0.072

0.073

0.074

0.075
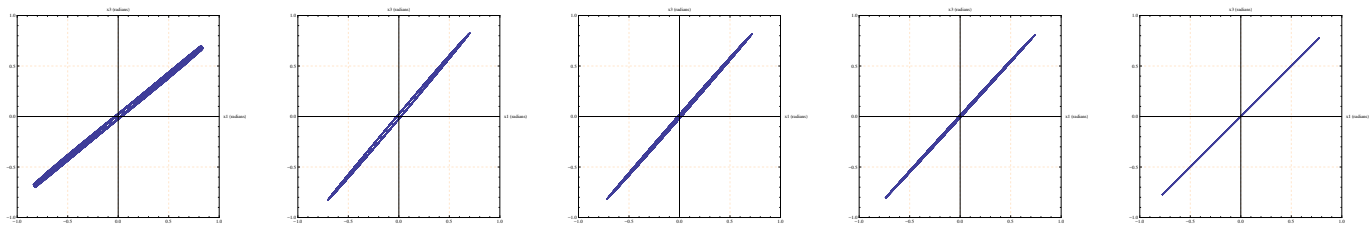

0.076

0.077

0.078

0.079

0.080

Fig. 18. The Lissajous figure for the coupling parameter values from 0.056 to 0.080. This shows the transition from anti-phase to in-phase synchronization. 


\section{Synchronization on a Passive Base}

The metronomes were set to various frequencies and then located on the passive base. The phase differences were observed for each frequency. Higher frequencies of metronomes correspond to more consumption of energy and more movements of the passive base (if any). The experimental results are shown in Figures 19 through 26.

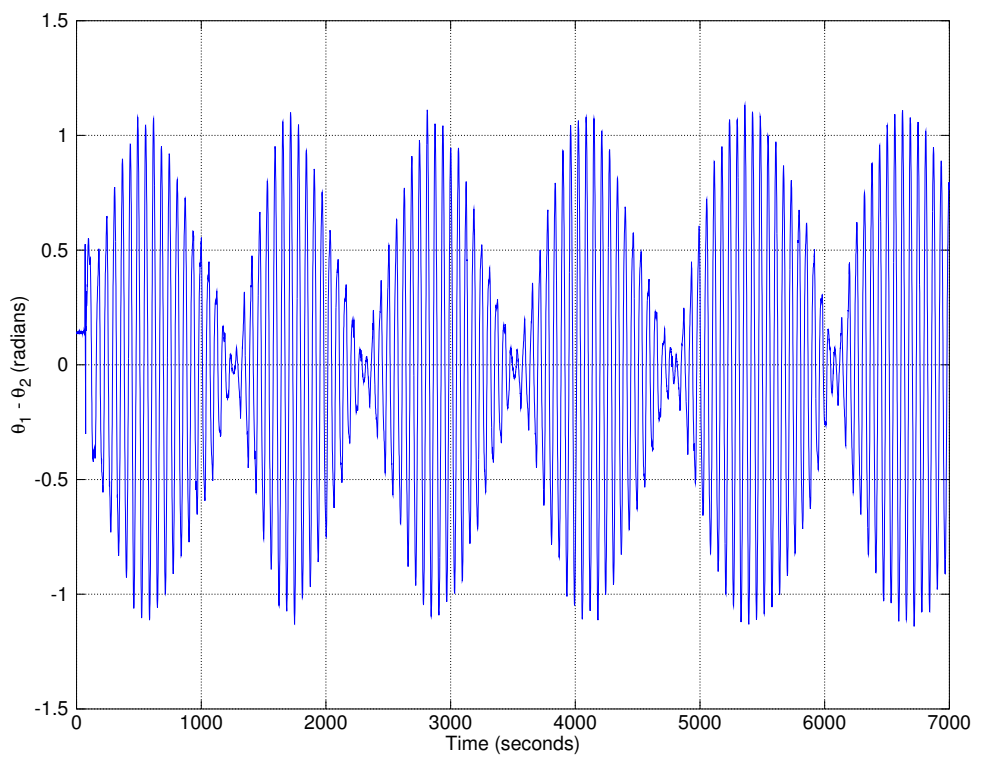

Fig. 19. Phase difference of two metronomes on a passive base $f_{1}=f_{2}=0.4 H z$ (which corresponds to 48 beats per minute) 


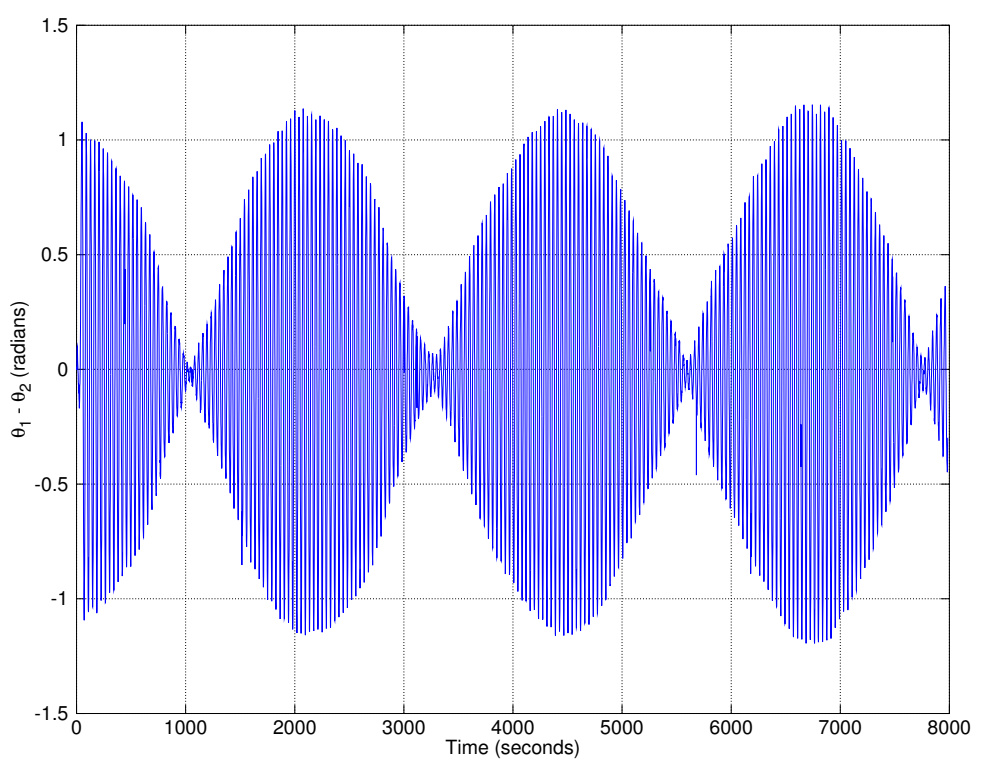

Fig. 20. Phase difference of two metronomes on a passive base $f_{1}=f_{2}=0.6 \mathrm{~Hz}$ (which corresponds to 72 beats per minute)

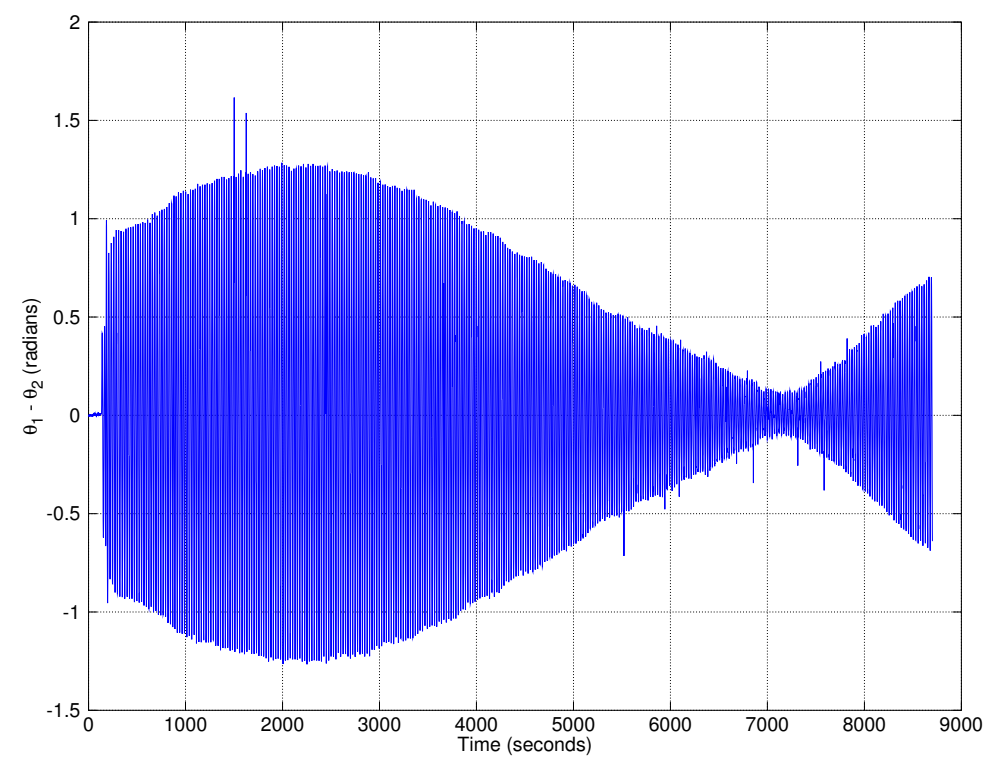

Fig. 21. Phase difference of two metronomes on a passive base $f_{1}=f_{2}=0.9 H z$ (which corresponds to 108 beats per minute) 


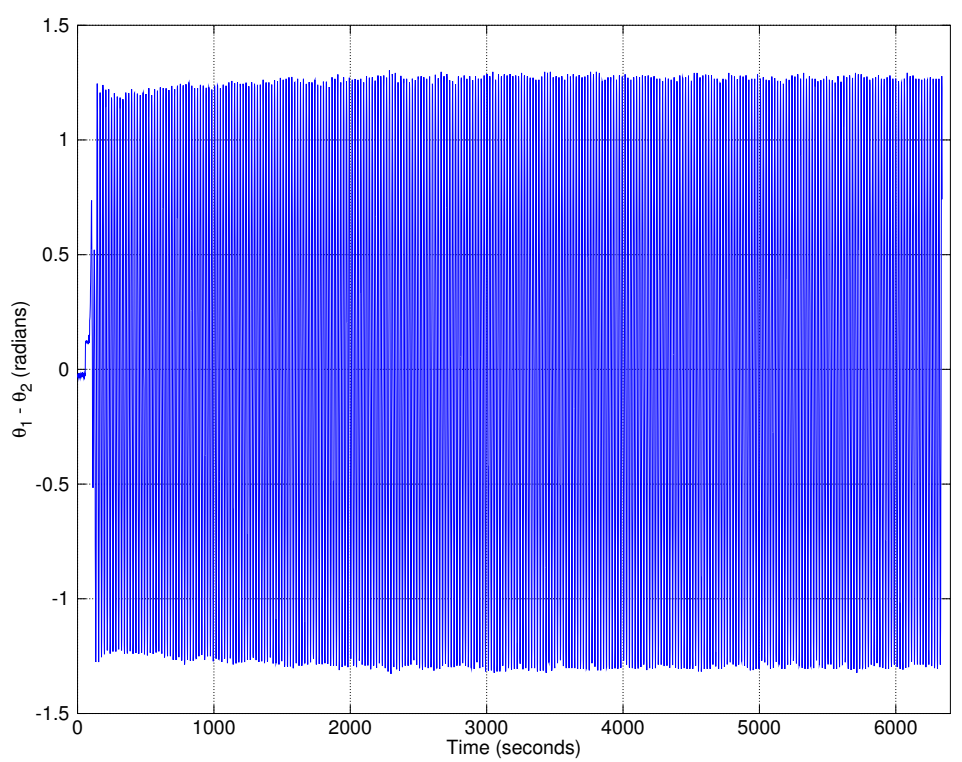

Fig. 22. Phase difference of two metronomes on a passive base $f_{1}=f_{2}=1.05 \mathrm{~Hz}$ (which corresponds to 126 beats per minute)

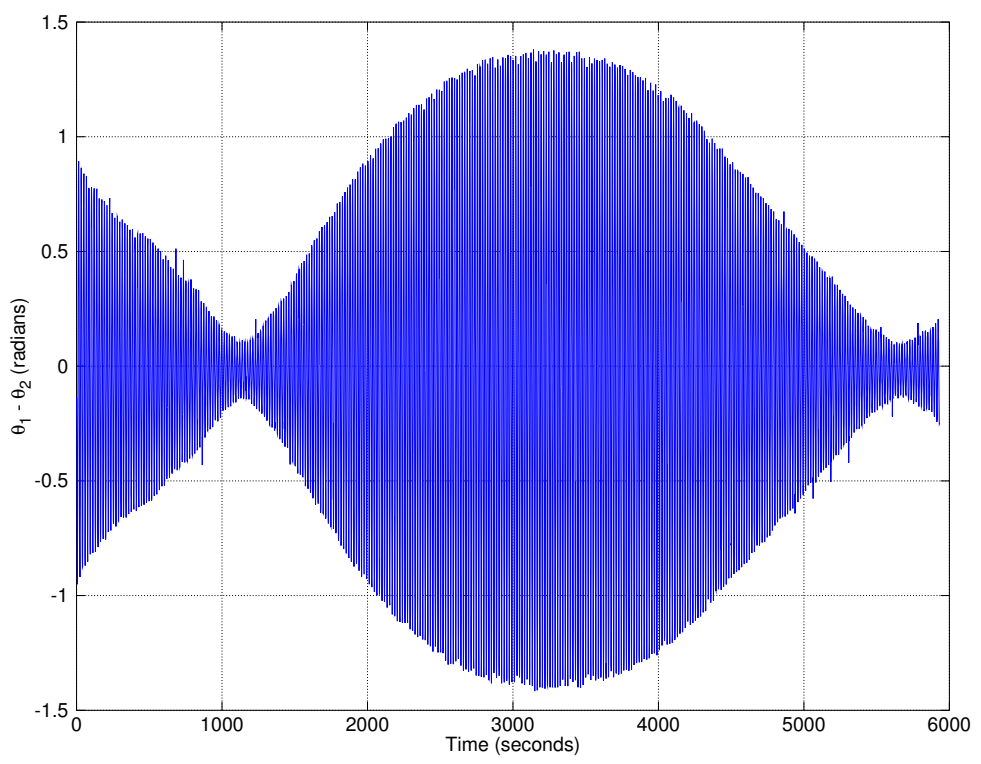

Fig. 23. Phase difference of two metronomes on a passive base $f_{1}=f_{2} \approx 1.27 \mathrm{~Hz}$ (which corresponds to 152 beats per minute) 


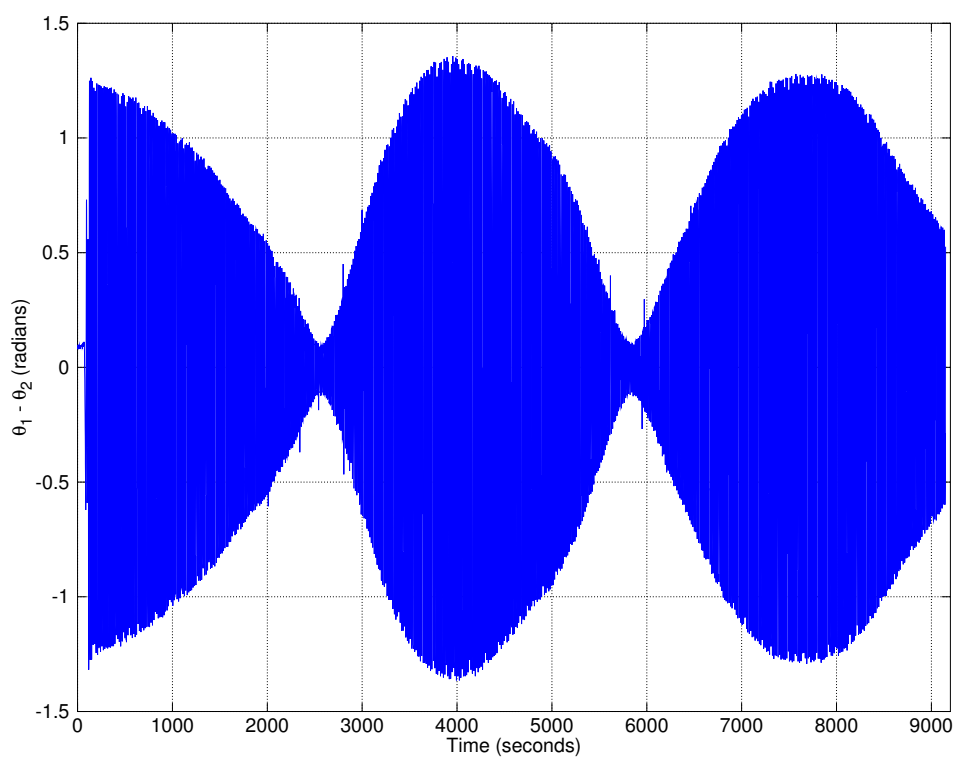

Fig. 24. Phase difference of two metronomes on a passive base $f_{1}=f_{2} \approx 1.47 \mathrm{~Hz}$ (which corresponds to 176 beats per minute)

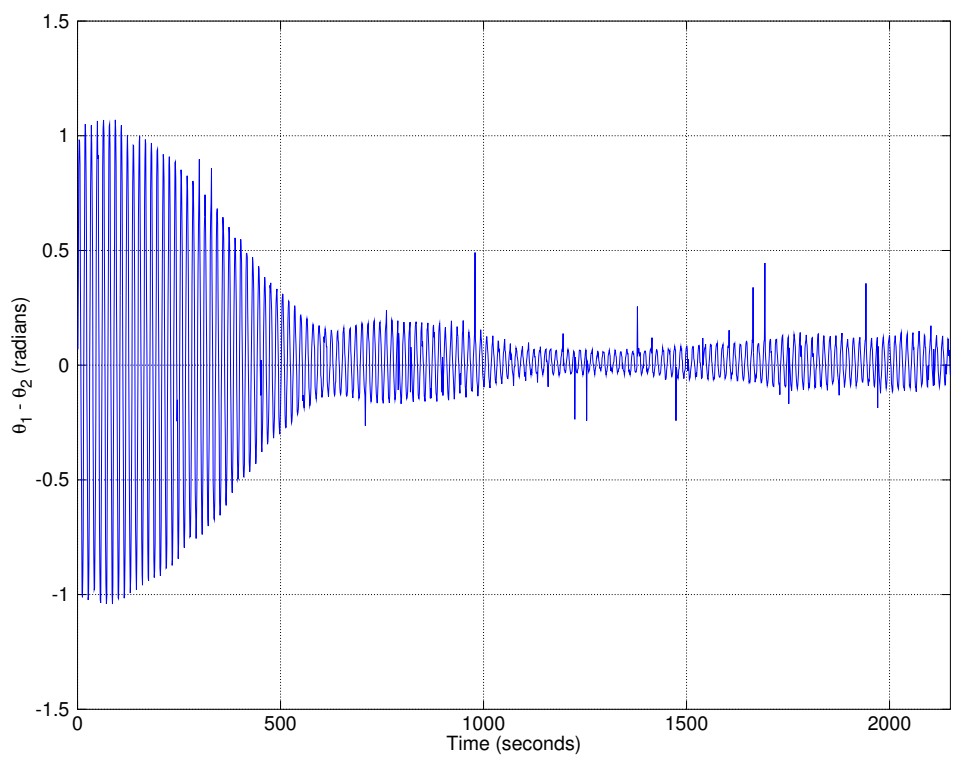

Fig. 25. Phase difference of two metronomes on a passive base $f_{1}=f_{2}=1.6 \mathrm{~Hz}$ (which corresponds to 192 beats per minute) 


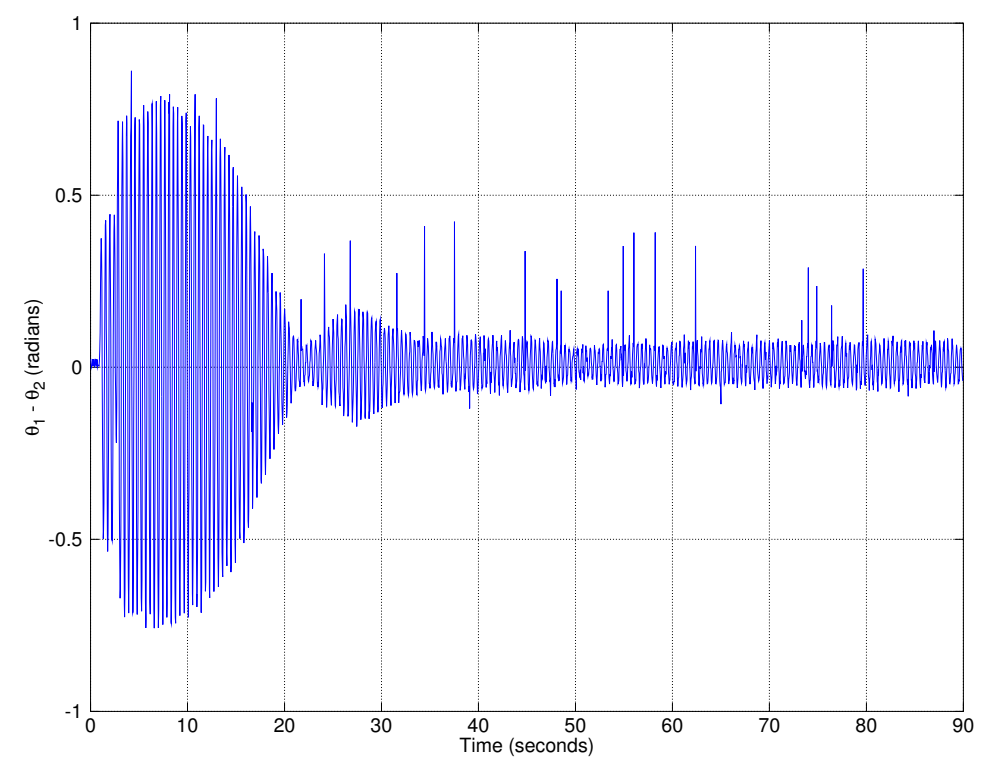

Fig. 26. Phase difference of two metronomes on a passive base $f_{1}=f_{2} \approx 1.73 \mathrm{~Hz}$ (which corresponds to 208 beats per minute) 
In an experiment the frequency of the metronomes were set to $0.4 \mathrm{~Hz}$ and they were located on a passive base. The base was a freely moving platform on two wheels. In Fig. 27 the distance of the detected pivot point from the regression line for the whole test is presented.

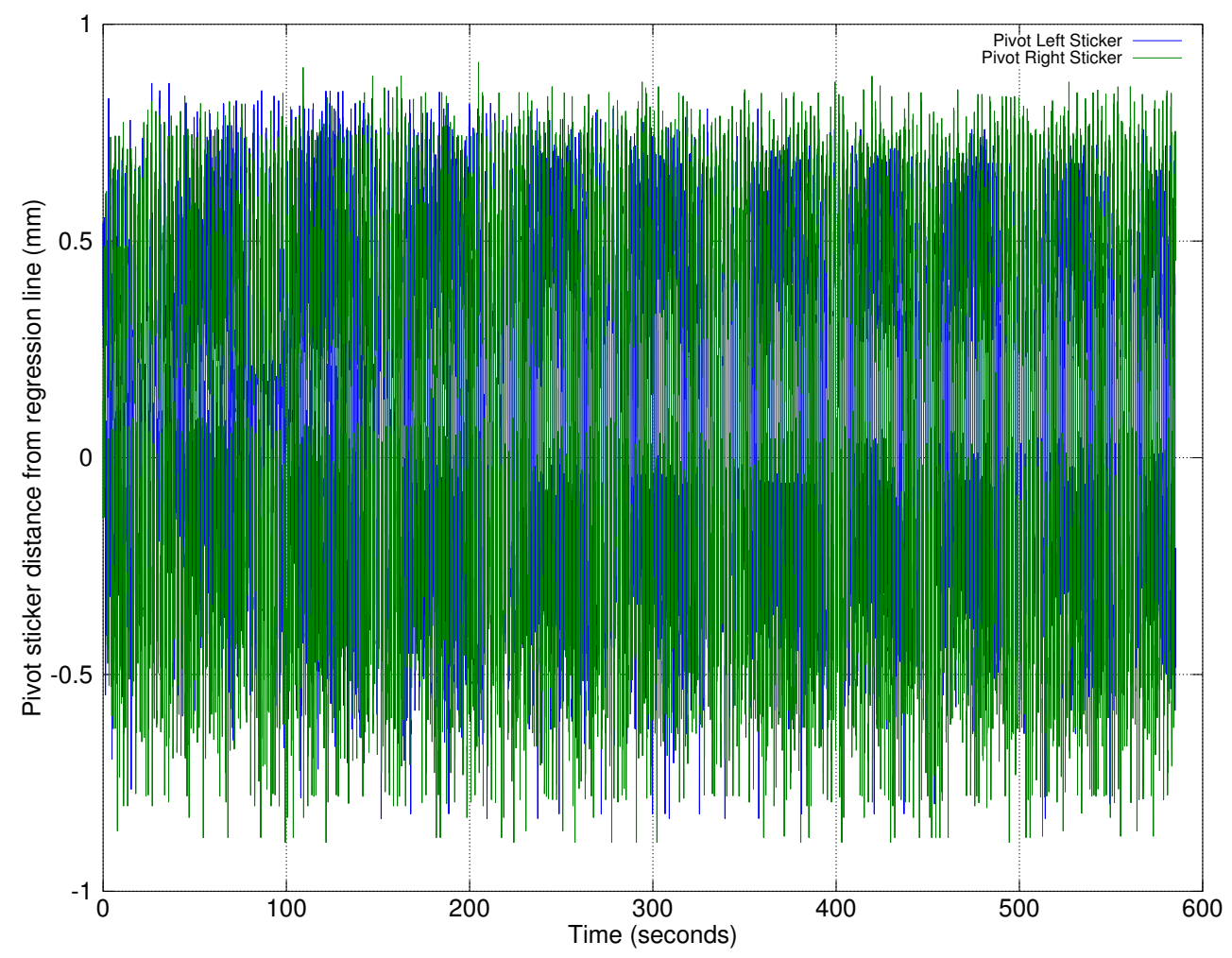

Fig. 27. The distances of the detected pivot points from the regression lines of all the stickers. The metronomes are located on a freely moving base and the frequencies are $0.4 \mathrm{~Hz}$ (which corresponds to 48 beats per minute).

\section{Conclusions}

In this chapter we discussed synchronization of oscillators. We described the model of our self sustained oscillators and provided more details about the effect of their 
parameters. We also showed the proposed model of metronomes when located on a freely moving base. We showed how the oscillations affect the base and on the other hand how the movements of the base affect each of the metronomes. We discussed the impact of base damping on the synchronization; whether the metronomes synchronize in-phase or anti-phase. At the end experimental results of synchronization of metronomes on a common base were shown. 


\section{CHAPTER IV}

\section{FORCED OSCILLATORS AND ADAPTATION}

In this chapter we investigate the effect of forcing the oscillators and will show how the system evolves in various forcing conditions.

\section{A. Forced Oscillators}

When the metronome base moves, we have a forced oscillator for which the governing equation is as below [23]

$$
\frac{d^{2} \theta}{d t^{2}}+\underbrace{\frac{m r_{c . m .} g}{I}}_{k} \sin (\theta)+\epsilon\left[\left(\frac{\theta}{\theta_{0}}\right)^{2}-1\right] \frac{d \theta}{d t}+\underbrace{\left(\frac{r_{c . m .} m}{I}\right)}_{l} \cos (\theta) \frac{d^{2} x}{d t^{2}}=0
$$

in which $x$ is the horizontal position of the base. We write the governing equation as below

$$
\begin{aligned}
& \frac{d \theta}{d t}=\gamma \\
& \frac{d \gamma}{d t}=-k \sin (\theta)-\epsilon\left[\left(\frac{\theta}{\theta_{0}}\right)^{2}-1\right] \gamma-l \cos (\theta) \frac{d^{2} x}{d t^{2}}
\end{aligned}
$$

When we form a feedback loop between the phase of the oscillator and the movements of the base, we have the following set of equations 


$$
\begin{aligned}
& \frac{d \theta}{d t}=\gamma \\
& \frac{d \gamma}{d t}=-k \sin (\theta)-\epsilon\left[\left(\frac{\theta}{\theta_{0}}\right)^{2}-1\right] \gamma-l \cos (\theta) \lambda \\
& \frac{d \lambda}{d t}=h(\theta, \gamma)
\end{aligned}
$$

in which

$$
\lambda=\frac{d^{2} x}{d t^{2}}
$$

and $h(\theta, \gamma)$ is a function specifying the feedback. This function depends on how the base responds to the detected angles of the metronome. For instance, $h(\theta, \gamma)=\theta$ means that the cart sets its acceleration to the same as the phase of the oscillator. A sample of trajectory for $\epsilon=0$ and $h(\theta, \gamma)=\theta$ is shown in Fig. 28 and a sample of trajectory for $\epsilon=0.2$ and $h(\theta, \gamma)=\theta$ is shown in Fig. 29 .

\section{B. Two Oscillators on an Active Base}

In this case we consider two metronomes located on a moving base. The metronomes oscillate freely while the movements of the base impacts their phase and frequencies. The equations of motion for this case are as follows 

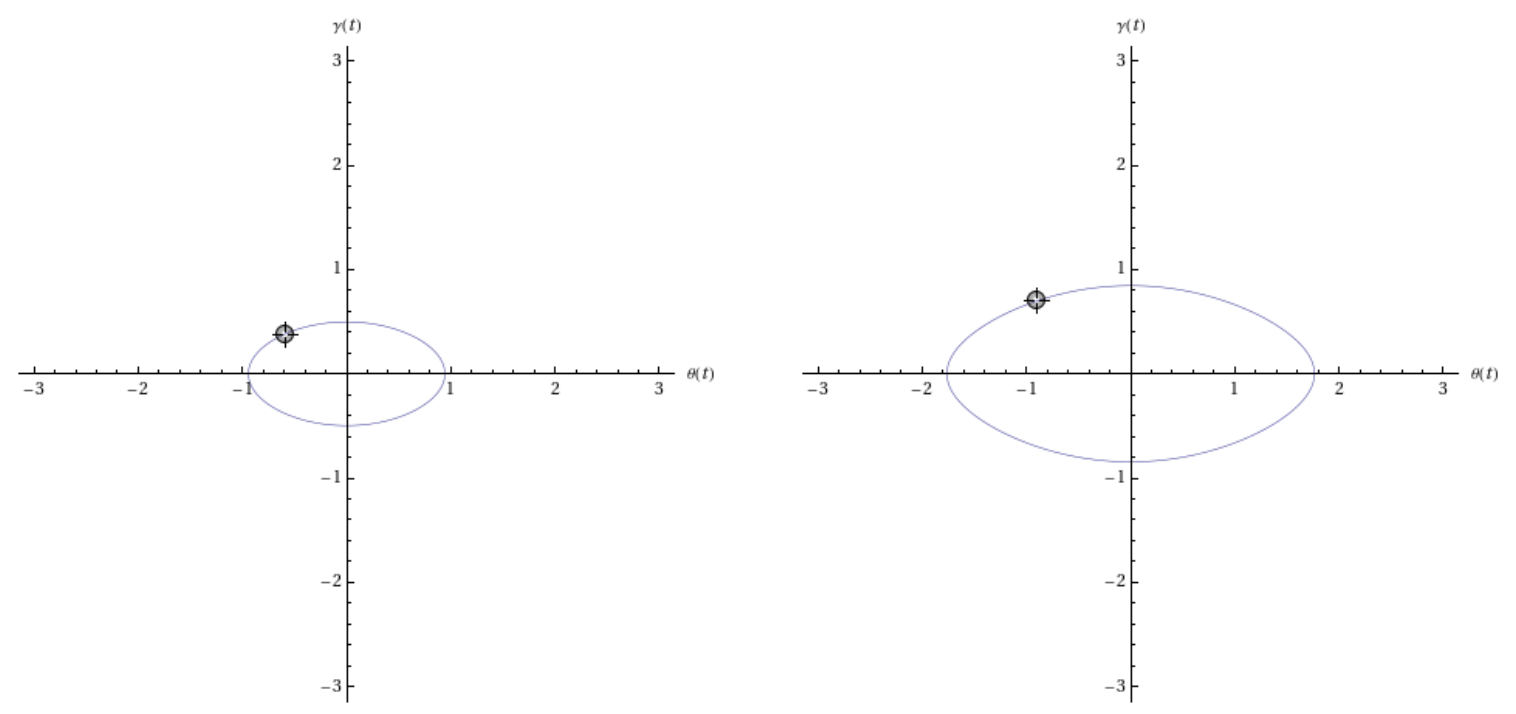

Fig. 28. The trajectory of the phase when the base moves with $\epsilon=0$. In this case $k=0.3, \epsilon=0$ and $\theta_{0}=\frac{\pi}{4}$

$$
\begin{aligned}
& \dot{x_{1}}=x_{2} \\
& \dot{x_{2}}=-\underbrace{\frac{m r_{c . m .} g}{I}}_{k} \sin \left(x_{1}\right)-\epsilon\left[\left(\frac{x_{1}}{\theta_{0}}\right)^{2}-1\right] x_{2}-\underbrace{\frac{m r_{c . m .}}{I}}_{l} \cos \left(x_{1}\right) x_{7} \\
& \dot{x_{3}}=x_{4} \\
& \dot{x_{4}}=-\underbrace{\frac{m r_{c . m . g}}{I}}_{k} \sin \left(x_{3}\right)-\epsilon\left[\left(\frac{x_{3}}{\theta_{0}}\right)^{2}-1\right] x_{4}-\underbrace{\frac{m r_{c . m .}}{I}}_{l} \cos \left(x_{3}\right) x_{7} \\
& \dot{x_{7}}=h\left(x_{1}, x_{2}, x_{3}, x_{4}\right)
\end{aligned}
$$

in which $x_{1}$ and $x_{3}$ are the angles the metronomes make with the vertical and $x_{2}$ and $x_{4}$ are their derivatives (i.e. rotational speeds). $x_{5}$ is the horizontal position of the base, $x_{6}=\dot{x}_{5}$ is the linear velocity of the base and $x_{7}=\dot{x}_{6}$ is the acceleration of the base 

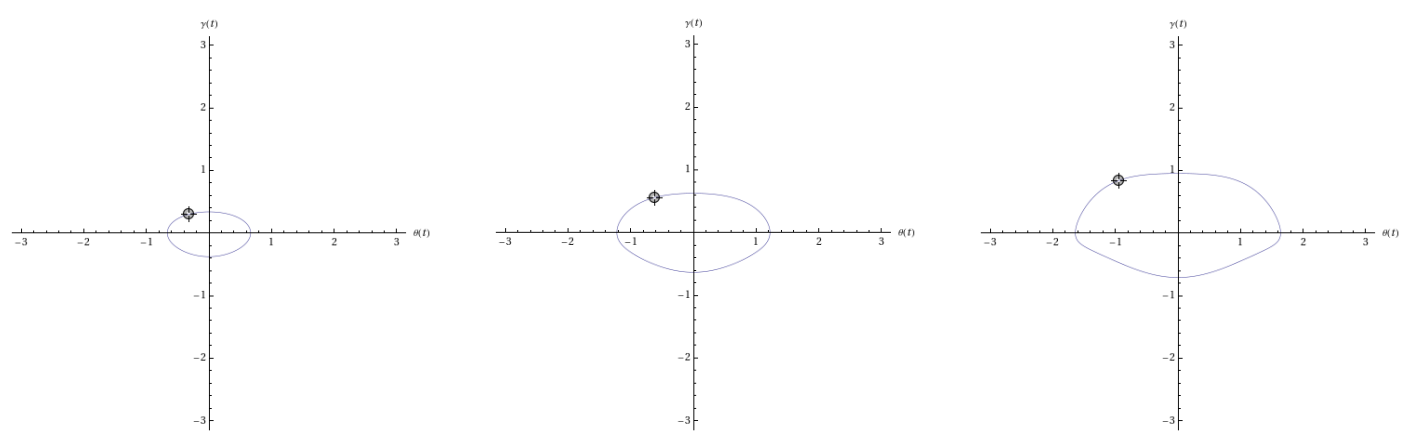

Fig. 29. The trajectory of the phase when the base moves with $\epsilon \neq 0$. In this case $k=0.3, \epsilon=0.2$ and $\theta_{0}=\frac{\pi}{4}$

(we have only included $x_{7}$ because it is the only parameter affecting the oscillators). $I$ is the moment of inertia of the pendulum, $\mathrm{m}$ is the mass of the pendulum, $r_{c . m}$. is the distance of the pendulums center of mass from the pivot point and $g$ is the acceleration of gravity. We now consider two sample functions for $h\left(x_{1}, x_{2}, x_{3}, x_{4}\right)$ in Equation $4.8 \mathrm{e}$ and study the impact of these two functions on the oscillators.

1. Case 1: $h\left(x_{1}, x_{2}, x_{3}, x_{4}\right)=m \sin \left(x_{2}+x_{4}\right)$

In this case the equations of motions are

$$
\begin{aligned}
& \dot{x_{1}}=x_{2} \\
& \dot{x_{2}}=-k \sin \left(x_{1}\right)-\epsilon\left[\left(\frac{x_{1}}{\theta_{0}}\right)^{2}-1\right] x_{2}-l \cos \left(x_{1}\right) x_{7} \\
& \dot{x_{3}}=x_{4} \\
& \dot{x_{4}}=-k \sin \left(x_{3}\right)-\epsilon\left[\left(\frac{x_{3}}{\theta_{0}}\right)^{2}-1\right] x_{4}-l \cos \left(x_{3}\right) x_{7} \\
& \dot{x_{7}}=m \sin \left(x_{2}+x_{4}\right)
\end{aligned}
$$


in which $m$ is a coefficient specifying the relation between the values perceived from the metronomes and the actions to be performed by the active base. We use this value to partially adjust the behaviors of the base. In the implementation of the settings, this value is set in the controller code (which implements the relation between sensory values and actuator commands). To find out the fixed points of the above model, we have

$$
\dot{x_{1}}=0 \Rightarrow x_{2}=0
$$

and

$$
\begin{aligned}
\dot{x_{2}}=0 & \Rightarrow-k \sin \left(x_{1}\right)-\epsilon\left[\left(\frac{x_{1}}{\theta_{0}}\right)^{2}-1\right] x_{2}-l \cos \left(x_{1}\right) x_{7}=0 \\
& \Rightarrow-k \sin \left(x_{1}\right)-l \cos \left(x_{1}\right) x_{7}=0
\end{aligned}
$$

which leads to the following values for $x_{1}$

$$
\begin{aligned}
& x_{1}=-\cos ^{-1}\left(-\frac{k}{\sqrt{x_{7}^{2} l^{2}+k^{2}}}\right) \\
& x_{1}=\cos ^{-1}\left(-\frac{k}{\sqrt{x_{7}^{2} l^{2}+k^{2}}}\right) \\
& x_{1}=-\cos ^{-1}\left(\frac{k}{\sqrt{x_{7}^{2} l^{2}+k^{2}}}\right) \\
& x_{1}=\cos ^{-1}\left(\frac{k}{\sqrt{x_{7}^{2} l^{2}+k^{2}}}\right)
\end{aligned}
$$

we also have 


$$
\dot{x_{3}}=0 \Rightarrow x_{4}=0
$$

and

$$
\begin{aligned}
\dot{d}=0 & \Rightarrow-k \sin \left(x_{3}\right)-\epsilon\left[\left(\frac{x_{3}}{\theta_{0}}\right)^{2}-1\right] d-l \cos \left(x_{3}\right) x_{7}=0 \\
& \Rightarrow-k \sin \left(x_{3}\right)-l \cos \left(x_{3}\right) x_{7}=0
\end{aligned}
$$

which leads to the following values for $c$

$$
\begin{aligned}
& x_{3}=-\cos ^{-1}\left(-\frac{k}{\sqrt{x_{7}^{2} l^{2}+k^{2}}}\right) \\
& x_{3}=\cos ^{-1}\left(-\frac{k}{\sqrt{x_{7}^{2} l^{2}+k^{2}}}\right) \\
& x_{3}=-\cos ^{-1}\left(\frac{k}{\sqrt{x_{7}^{2} l^{2}+k^{2}}}\right) \\
& x_{3}=\cos ^{-1}\left(\frac{k}{\sqrt{x_{7}^{2} l^{2}+k^{2}}}\right)
\end{aligned}
$$

as $x_{2}=x_{4}=0$, we have

$$
\dot{x}_{7}=0
$$

So, for specific values of $k$ and $l$ and a specific initial value of $x_{7}$, we have 16 fixed points in the system. 
2. Case 2: $h\left(x_{1}, x_{2}, x_{3}, x_{4}\right)=m \sin \left(x_{1}+x_{3}\right)$

In this case the equations of motions are

$$
\begin{aligned}
& \dot{x_{1}}=x_{2} \\
& \dot{x_{2}}=-k \sin \left(x_{1}\right)-\epsilon\left[\left(\frac{x_{1}}{\theta_{0}}\right)^{2}-1\right] x_{2}-l \cos \left(x_{1}\right) x_{7} \\
& \dot{x_{3}}=x_{4} \\
& \dot{x_{4}}=-k \sin \left(x_{3}\right)-\epsilon\left[\left(\frac{x_{3}}{\theta_{0}}\right)^{2}-1\right] d-l \cos \left(x_{3}\right) x_{7} \\
& \dot{x_{7}}=m \sin \left(x_{1}+x_{3}\right)
\end{aligned}
$$

in which $m$ is a coefficient as previously described. To find out the fixed points of the above model, we have

$$
\dot{x_{1}}=0 \Rightarrow x_{2}=0
$$

and

$$
\begin{aligned}
\dot{x_{2}}=0 & \Rightarrow-k \sin \left(x_{1}\right)-\epsilon\left[\left(\frac{x_{1}}{\theta_{0}}\right)^{2}-1\right] x_{2}-l \cos \left(x_{1}\right) x_{7}=0 \\
& \Rightarrow-k \sin \left(x_{1}\right)-l \cos \left(x_{1}\right) x_{7}=0
\end{aligned}
$$

which leads to the following values for $a$ 


$$
\begin{aligned}
& x_{1}=-\cos ^{-1}\left(-\frac{k}{\sqrt{x_{7}^{2} l^{2}+k^{2}}}\right) \\
& x_{1}=\cos ^{-1}\left(-\frac{k}{\sqrt{x_{7}^{2} l^{2}+k^{2}}}\right) \\
& x_{1}=-\cos ^{-1}\left(\frac{k}{\sqrt{x_{7}^{2} l^{2}+k^{2}}}\right) \\
& x_{1}=\cos ^{-1}\left(\frac{k}{\sqrt{x_{7}^{2} l^{2}+k^{2}}}\right)
\end{aligned}
$$

we also have

$$
\dot{x_{3}}=0 \Rightarrow x_{4}=0
$$

and

$$
\begin{aligned}
\dot{x_{4}}=0 & \Rightarrow-k \sin \left(x_{3}\right)-\epsilon\left[\left(\frac{x_{3}}{\theta_{0}}\right)^{2}-1\right] x_{4}-l \cos \left(x_{3}\right) x_{7}=0 \\
& \Rightarrow-k \sin \left(x_{3}\right)-l \cos \left(x_{3}\right) x_{7}=0
\end{aligned}
$$

which leads to the following values for $c$ 


$$
\begin{aligned}
& x_{3}=-\cos ^{-1}\left(-\frac{k}{\sqrt{x_{7}^{2} l^{2}+k^{2}}}\right) \\
& x_{3}=\cos ^{-1}\left(-\frac{k}{\sqrt{x_{7}^{2} l^{2}+k^{2}}}\right) \\
& x_{3}=-\cos ^{-1}\left(\frac{k}{\sqrt{x_{7}^{2} l^{2}+k^{2}}}\right) \\
& x_{3}=\cos ^{-1}\left(\frac{k}{\sqrt{x_{7}^{2} l^{2}+k^{2}}}\right)
\end{aligned}
$$

we also have

$$
\begin{aligned}
\dot{x_{7}}=0 & \Rightarrow m \sin \left(x_{1}+x_{3}\right)=0 \\
& \Rightarrow x_{1}+x_{3}=n \pi
\end{aligned}
$$

As the angles of the metronomes do not exceed a certain value, the only possible value for $n$ in the above formula is $n=0$. So we have

$$
x_{1}+x_{3}=0 \Rightarrow x_{1}=-x_{3}
$$

C. Two Oscillators on an Active Base with Adjusting Parameter

In this case we also consider two metronomes located on a moving base, but we introduce a parameter for changing the behavior of the system. The metronomes oscillate freely while the movements of the base impact their phase and frequencies. The movements of the base are a function of state variables with adjustments from a parameter. The equations of motion for this case are as follows 


$$
\begin{aligned}
& \dot{x_{1}}=x_{2} \\
& \dot{x_{2}}=-\underbrace{\frac{m r_{c . m . g}}{I}}_{k} \sin \left(x_{1}\right)-\epsilon\left[\left(\frac{x_{1}}{\theta_{0}}\right)^{2}-1\right] x_{2}-\underbrace{\frac{m r_{c . m .}}{I}}_{l} \cos \left(x_{1}\right) x_{7} \\
& \dot{x_{3}}=x_{4} \\
& \dot{x_{4}}=-\underbrace{\frac{m r_{c . m .}}{I}}_{k} \sin \left(x_{3}\right)-\epsilon\left[\left(\frac{x_{3}}{\theta_{0}}\right)^{2}-1\right] x_{4}-\underbrace{\frac{m r_{c . m .}}{I}}_{l} \cos \left(x_{3}\right) x_{7} \\
& \dot{x_{5}}=x_{6} \\
& \dot{x_{6}}=x_{7} \\
& \dot{x_{7}}=x_{9} x_{1}+\left(1-x_{9}\right) x_{3} \\
& \dot{x_{9}}=f\left(x_{1}, x_{2}, x_{3}, x_{4}, x_{7}, x_{9}\right)
\end{aligned}
$$

in which $f\left(x_{1}, x_{2}, x_{3}, x_{4}, x_{7}, x_{9}\right)$ in Equation $4.41 \mathrm{~h}$ is a low pass filter for modifying the adjusting parameter $\left(x_{9}\right)$. A sample function for $f$ is

$$
\dot{x_{9}}=f\left(x_{1}, x_{2}, x_{3}, x_{4}, x_{7}, x_{9}\right)=x_{9}-\underbrace{\left(-0.9 x_{9}+0.9 x_{1}\right)}_{\text {Low pass filter }}
$$




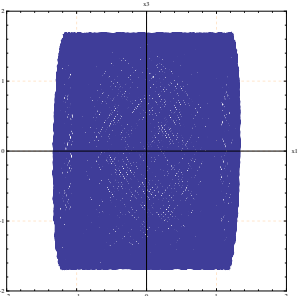

0.00

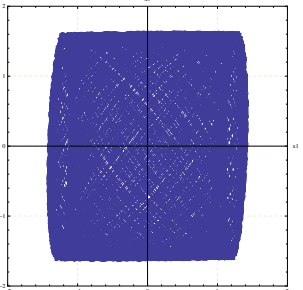

0.20

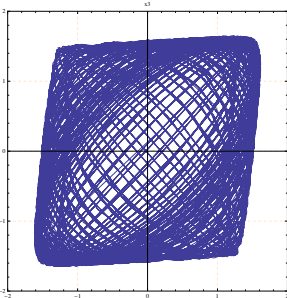

0.40

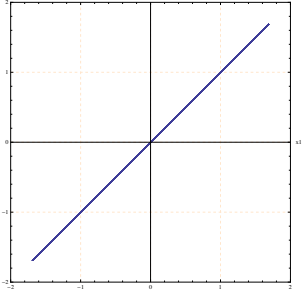

0.60

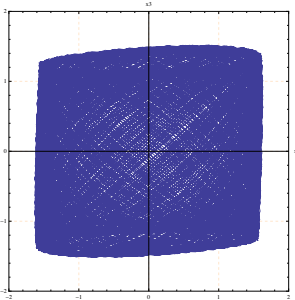

0.80

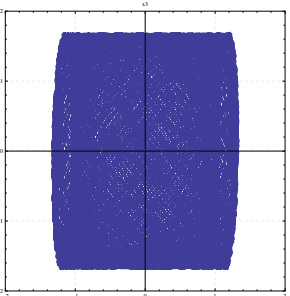

0.05

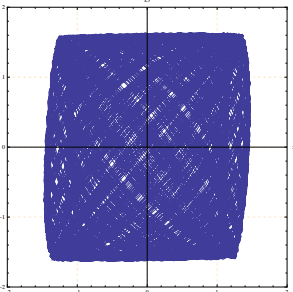

0.25

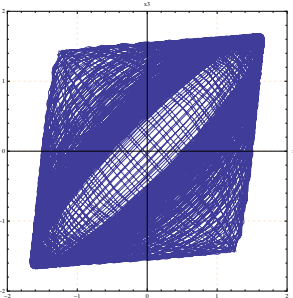

0.45

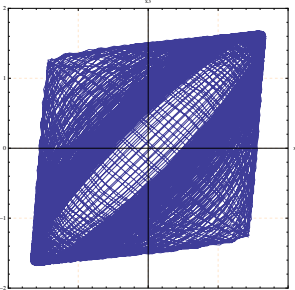

0.65

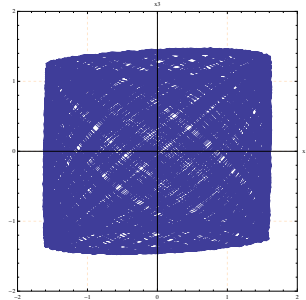

0.85

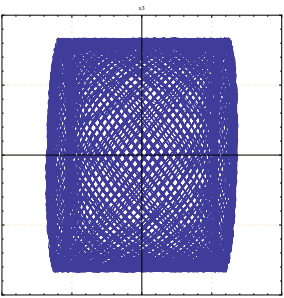

0.10

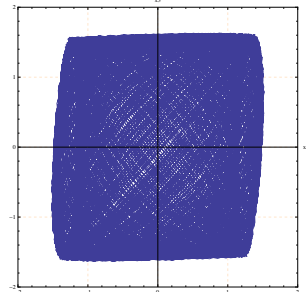

0.30

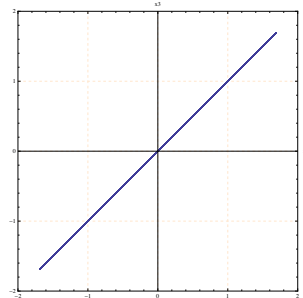

0.50

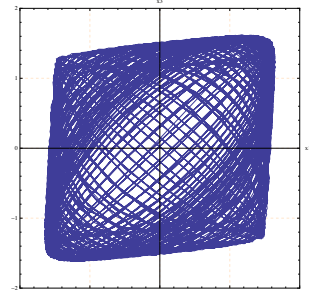

0.70

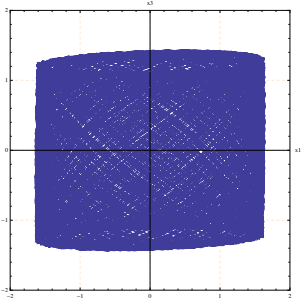

0.90

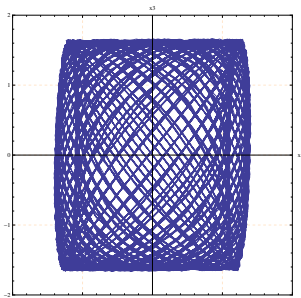

0.15

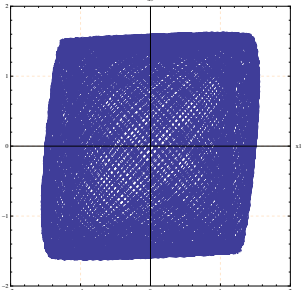

0.35

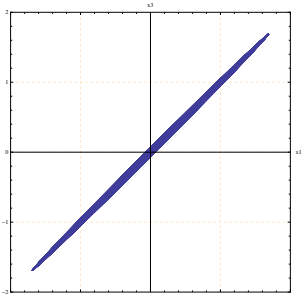

0.55

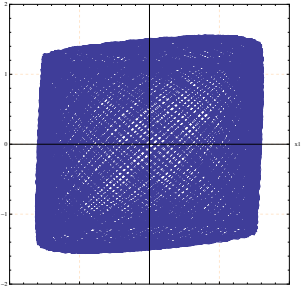

0.75

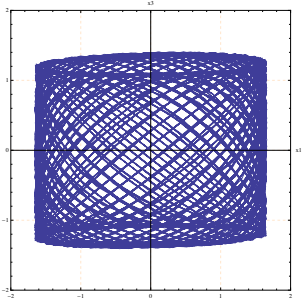

0.95

Fig. 30. Two metronomes on an actively moving base in which $\dot{x}_{7}=($ ratio $) \theta_{1}+(1-$ ratio $) \theta_{2}$. Shown in pictures are $\theta_{1}$ vs $\theta_{2}$ when transient is relaxed for various values of ratio. 


\section{Low-Pass Filtered Feedback}

A low-pass filter is made to provide feedback. The feedback is used for adjusting the parameter of the system which specifies the qualitative behavior of the system. The equivalent differential equation is written as

$$
R_{2} C \frac{d V_{\text {out }}}{d t}+V_{\text {out }}=-\frac{V_{\text {in }} R_{2}}{R_{1}}
$$

So, for our filter, we write the differential equation as follows

$$
\frac{d V_{\text {out }}}{d t}=\frac{1}{R_{2} C}\left(-\frac{V_{\text {in }} R_{2}}{R_{1}}-V_{\text {out }}\right)
$$

In this filter the cutoff frequency (in hertz) is defined as

$$
f_{c}=\frac{1}{2 \pi R_{2} C}
$$

or equivalently (in radians per second):

$$
\omega_{c}=\frac{1}{R_{2} C}
$$

The gain in the passband is $\frac{R_{2}}{R_{1}}$, and the stop-band drops off at $6 \mathrm{~dB}$ per octave as it is a first-order filter. Hence, the differential equations of our system will be as follows 


$$
\begin{aligned}
& \dot{x_{1}}=x_{2} \\
& \dot{x_{2}}=-\underbrace{\left(\frac{m r_{c . m .} g}{I}\right)}_{k} \sin \left(x_{1}\right)-\epsilon\left[\left(\frac{x_{1}}{\theta_{0}}\right)^{2}-1\right] x_{2}-\underbrace{\left(\frac{m r_{c . m .}}{I}\right)}_{l} \cos \left(x_{1}\right) x_{7} \\
& \dot{x_{3}}=x_{4} \\
& \dot{x_{4}}=-\underbrace{\left(\frac{m r_{c . m .}}{I}\right)}_{k} \sin \left(x_{3}\right)-\epsilon\left[\left(\frac{x_{3}}{\theta_{0}}\right)^{2}-1\right] x_{4}-\underbrace{\left(\frac{m r_{c . m .}}{I}\right)}_{l} \cos \left(x_{3}\right) x_{7} \\
& \dot{x_{7}}=-x_{9} x_{1}+\left(1-\left(-x_{9}\right)\right) x_{3} \\
& \dot{x_{9}}=\alpha\left(\frac{1}{R_{2} C}\right)\left(-x_{9}-\frac{x_{2} x_{4} R_{2}}{R_{1}}\right)
\end{aligned}
$$

In the filter, the feedback is attenuated based on [52]. Also the gain of the filter is negative (set to be -1 for this case) and hence the negated value of $x_{9}$ is used in the differential equations.

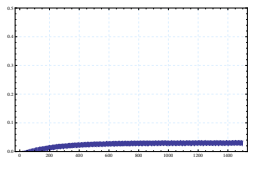

0.00

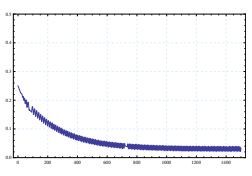

0.25

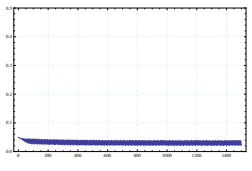

0.05

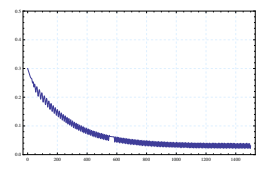

0.30

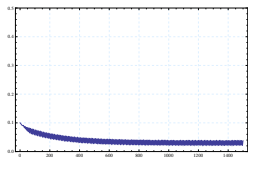

0.10

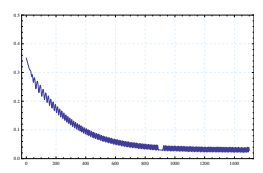

0.35

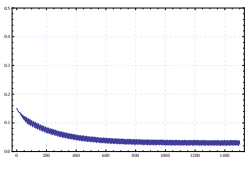

0.15

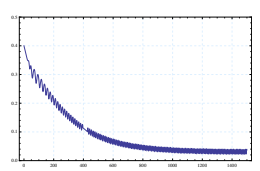

0.40

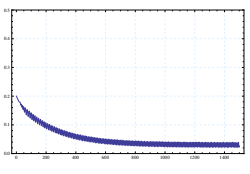

0.20

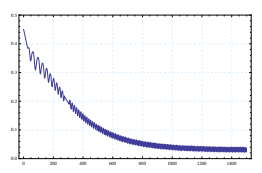

0.45

Fig. 31. Value of $x_{9}$ (the adjustable parameter) in time for initial values from 0.00 to 0.45 . 


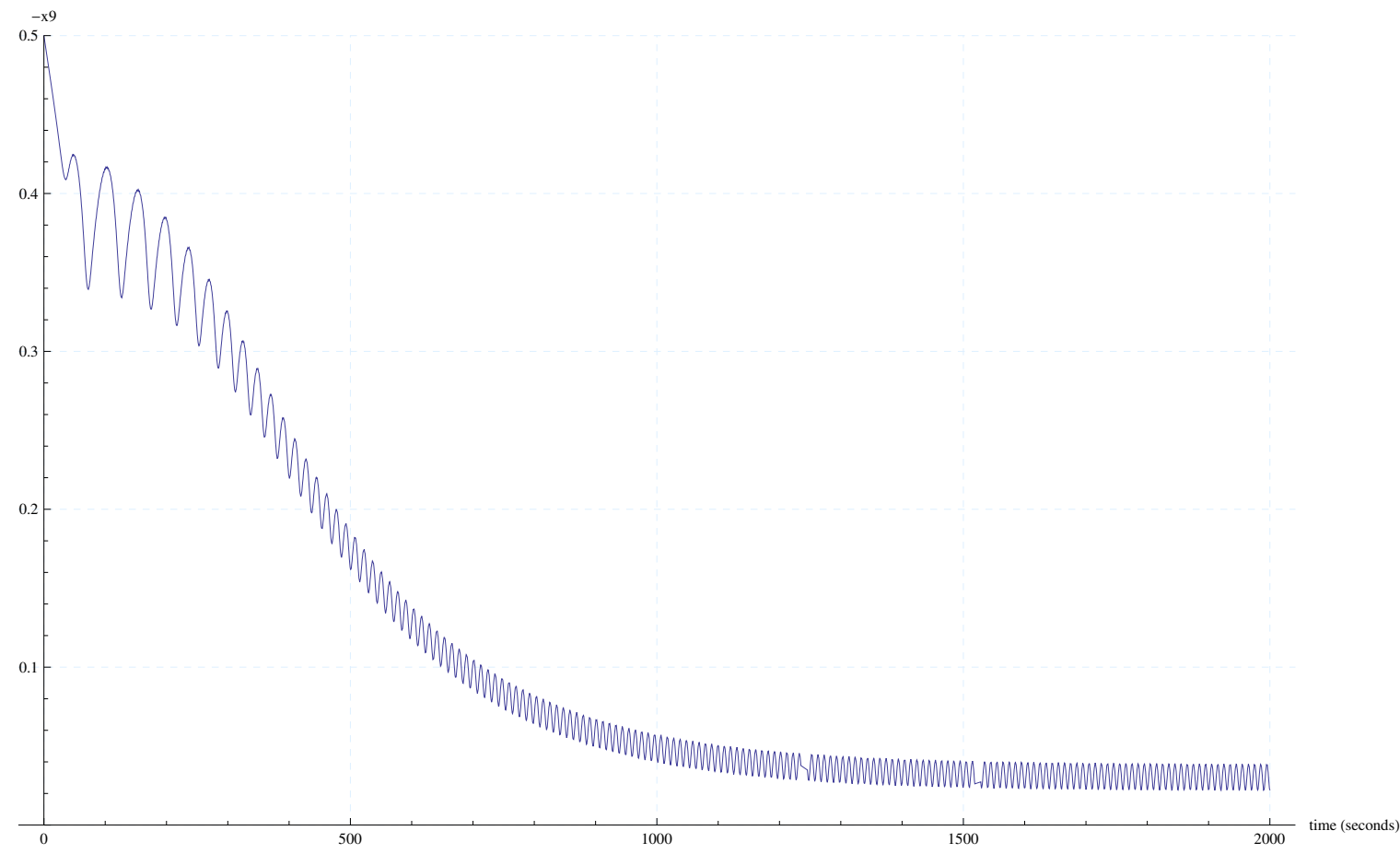

Fig. 32. Value of $x_{9}$ (the adjustable parameter) in time for initial value $=0.50$. 


\section{E. Adaptation in Experiments}

In another experiment the tests were set such that the speed of the cart (and not the acceleration) was changed as a function of the angles of metronomes. In this case we have

$$
x_{6}=\epsilon\left(x_{9} x_{1}+\left(1-x_{9}\right) x_{3}\right)
$$

in which $\epsilon$ is an attenuating factor. The effect of $x_{9}$ parameter without adjustment is shown in Figures 33 and 34 and the phase difference and the value of the adjusting parameter evolving in time are shown in Fig. 35.

\section{F. Conclusions}

In this chapter we reviewed the model of forced oscillators. We discussed the model of a metronome located on a moving base and analyzed the effects of the base movements on the metronome. We described the case in which two metronomes are located on a common base and the movements of the base are a function of a feedback from their state variables. We analyzed the behavior of the system for some sample functions of the feedback. We then introduced an adjusting parameter which determines the feedback. The feedback receives input from state variables and provides output for the movements of the common base. We showed the effect of the parameter on the behavior of the system and then let it change. We showed that how the adjusting parameter evolves through time when it changes according to a low-pass filtered feedback from some state variables of the system. The results were supported with results from experiments. 


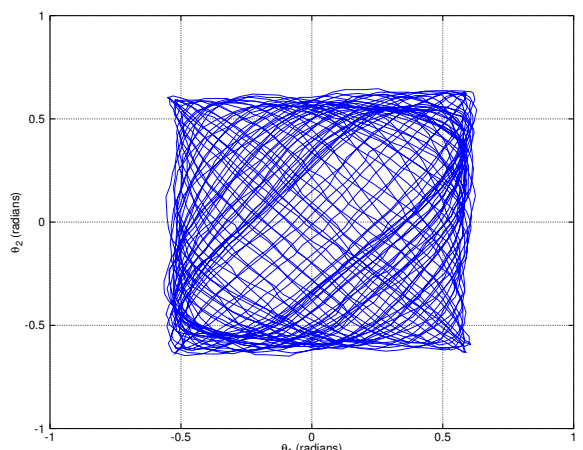

0.25

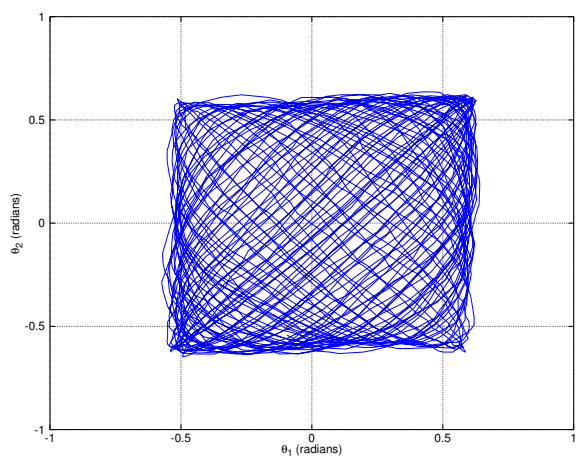

0.35

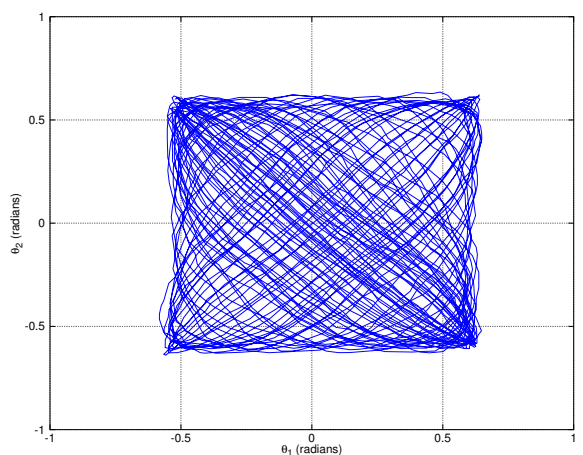

0.45

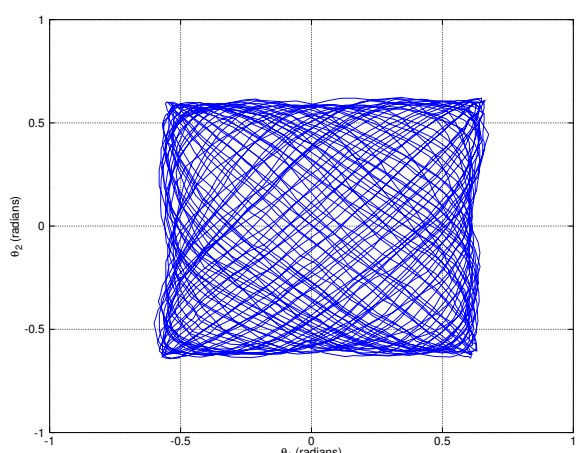

0.30

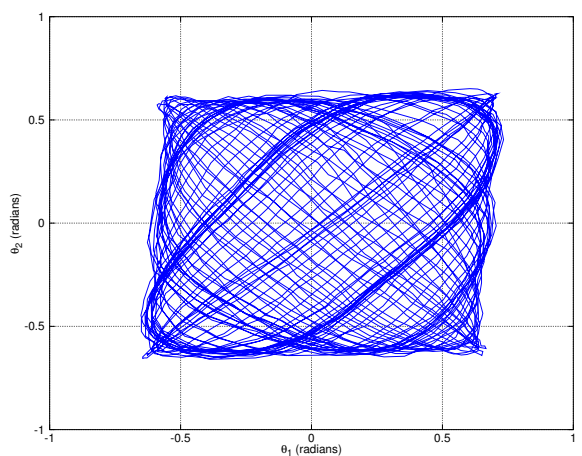

0.40

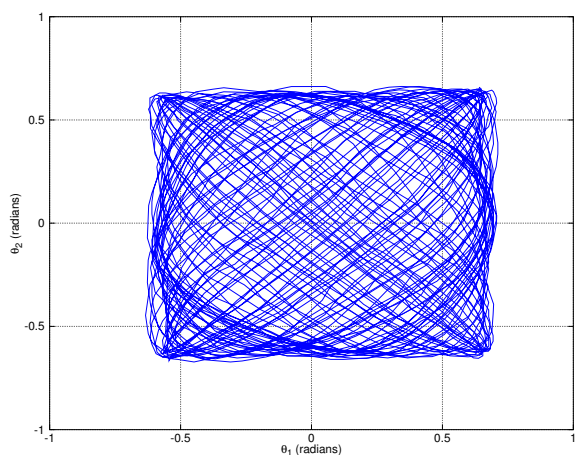

0.50

Fig. 33. The Lissajous figures of phases of metronomes in experiment. The transients are relaxed and $\epsilon=0.3$. The parameter $x_{9}$ is fixed and the values are 0.25 to 0.50. The metronomes are located on a moving cart and their frequencies of oscillations are $0.4 \mathrm{~Hz}$ (which corresponds to 48 beats per minute). 


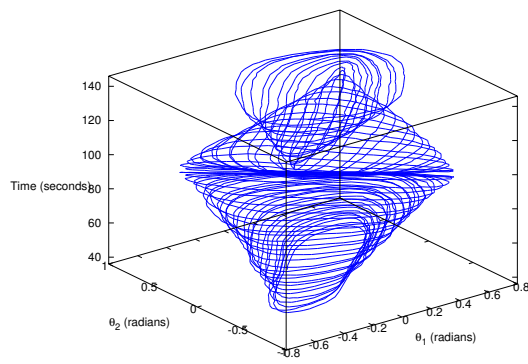

0.25

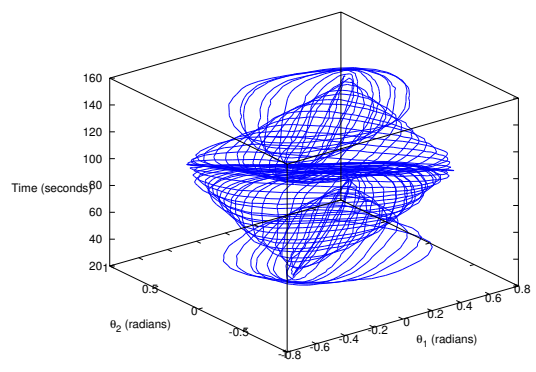

0.35

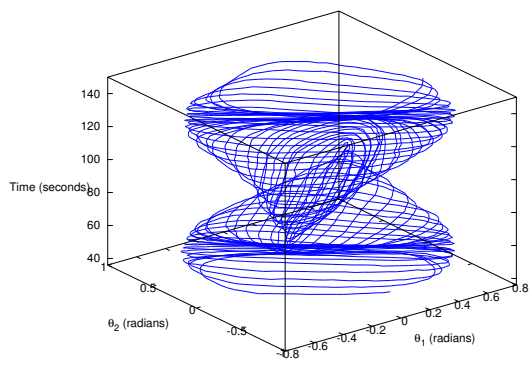

0.45

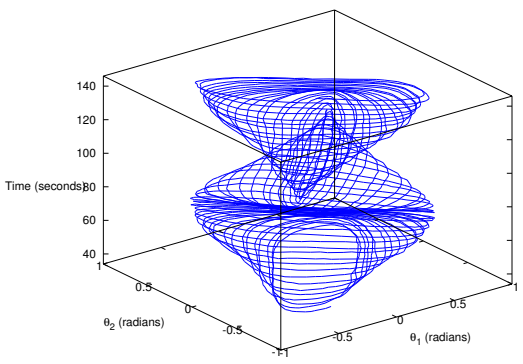

0.30

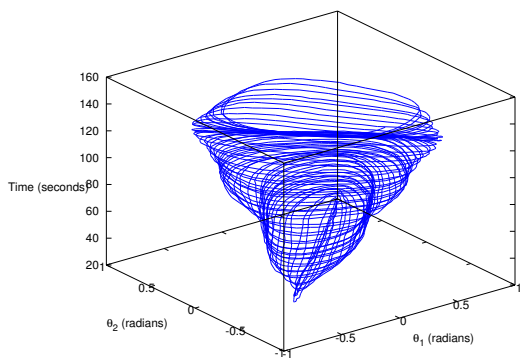

0.40

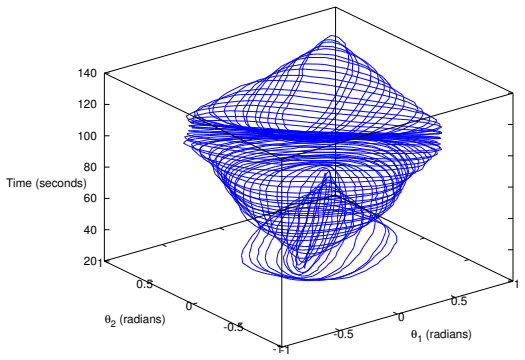

0.50

Fig. 34. The Lissajous figures of phases of metronomes through time in experiment. The transients are relaxed and $\epsilon=0.3$. The parameter $x_{9}$ is fixed and the values are 0.25 to 0.50 . The metronomes are located on a moving cart and their frequencies of oscillations are $0.4 \mathrm{~Hz}$ (which corresponds to 48 beats per minute). 

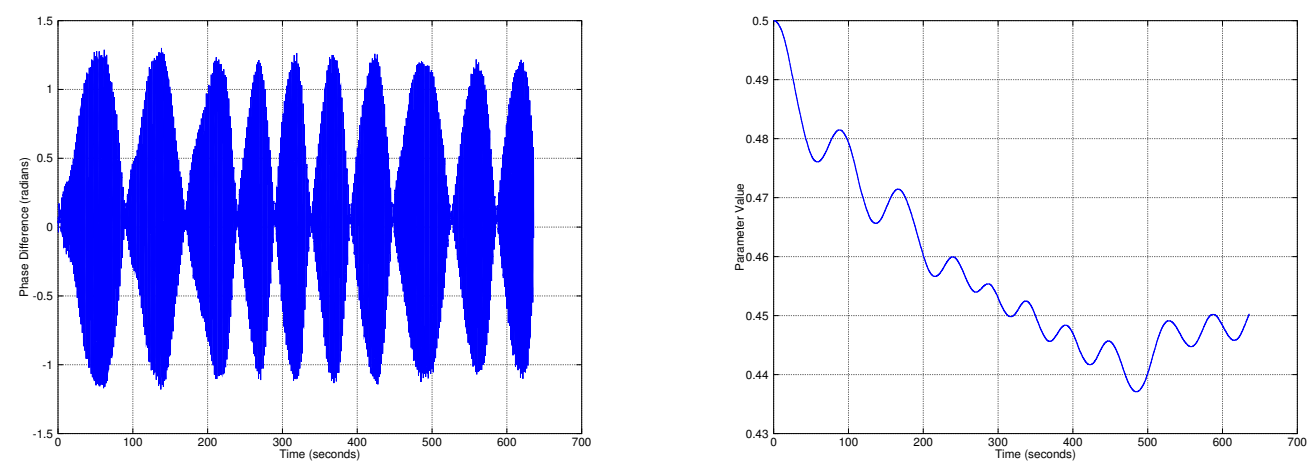

$$
\alpha=0.001, R_{1}=R_{2}, R_{1} C=2.0
$$

$$
\alpha=0.001, R_{1}=R_{2}, R_{1} C=2.0
$$
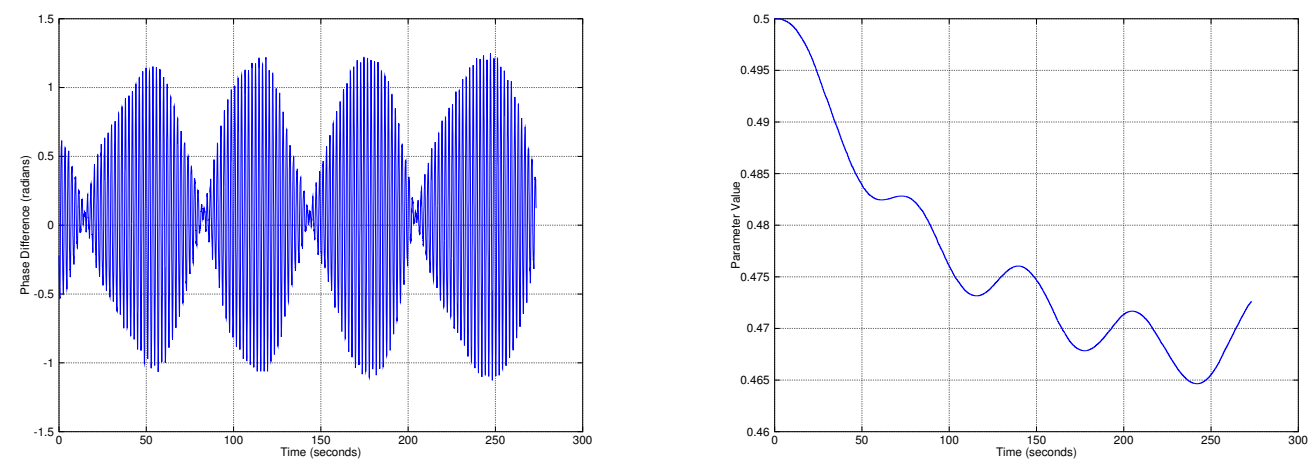

$\alpha=0.005, R_{1}=R_{2}, R_{1} C=2.0$

$$
\alpha=0.005, R_{1}=R_{2}, R_{1} C=2.0
$$
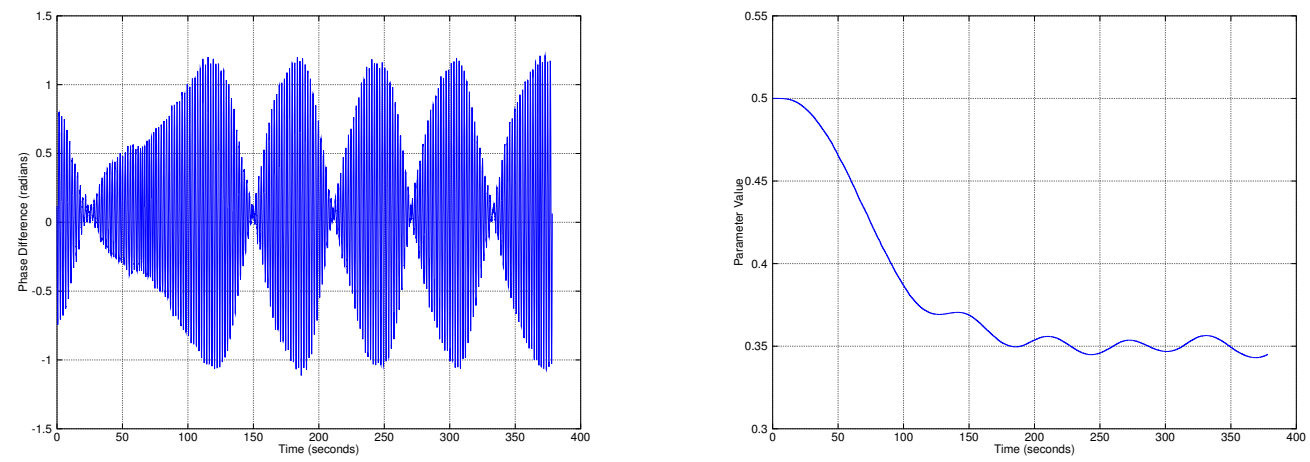

$\alpha=0.001, R_{1}=R_{2}, R_{1} C=2.0$

$$
\alpha=0.001, R_{1}=R_{2}, R_{1} C=2.0
$$

Fig. 35. The phase difference of metronomes $x_{1}-x_{3}$ and value of the adjustable parameter $x_{9}$ through time during experiments. The phase difference is shown on the left and the value of the adjustable parameter is shown on the right. $\epsilon=0.3$, Three stickers are used on the metronomes, the metronomes are located on a moving cart and their frequencies of oscillations are $0.4 \mathrm{~Hz}$ (which corresponds to 48 beats per minute). 


\section{CHAPTER V}

\section{CONCLUSIONS AND FUTURE WORK}

In this chapter we have conclusions of this research and will provide some recommendations for future research.

\section{A. Conclusions}

In this research we considered the synchronization between two oscillators that are mechanically coupled. We described the coupling possibility and showed some parameters that affected the coupling. The settings for the experiments were described and we showed how a simple vision system with a light-weight image processing algorithm can help to effectively find the phase of two oscillators. We demonstrated how the oscillators synchronize and what is the relationship between the oscillation frequency and the synchronization speed. We also showed how an active base affects the behavior of oscillators and when the system is adjustable, how this dynamical system evolves over time.

\section{B. Future Work}

There are some improvements that are suggested for the future work of this research.

\section{Model}

For analysis and numerical simulation, we considered an ideal model of the system in which there are not any delays or random terms. An area for future work would be to include these terms in the model and investigate the system. The similarity to the experimental results would show the accuracy of the model. 


\section{Experiments}

Using other alternatives for detecting the phases of the oscillators is a possible improvement for the system. This might include using sound detecting devices ${ }^{1}$ and encoders for detecting the angles of the metronomes ${ }^{2}$. Other devices for sensing can be used simultaneously so that the accuracy of the data is increased.

\section{Coupling}

The oscillators are mechanically coupled in this system. A possible direction of future research would be considering a case in which the coupling involves delay. This can be realized when the metronomes are positioned on two independent carts and each of the carts is capable of moving independently. The same can be done for when there are random terms in the coupling between oscillators.

\section{Oscillators}

A possible area for extending the current work would be to introduce more oscillators. The number of oscillators and their positioning besides how they are connected to each other are some sample parameters of the system to be determined. A higher number of oscillators will certainly bring more complexity to the system and we expect to see more complex (and maybe richer) behaviors. We would also recommend using other types of oscillators that are not necessarily mechanical oscillators (for instance electrical circuits). A similar system can be replicated with other oscillators and the evolution of the system can be studied.

\footnotetext{
${ }^{1}$ The metronomes make a tick sound when the rod reaches the far left or far right.

${ }^{2}$ Reading the values of the angles by using mechanical devices is acceptable if the sensory device does not interfere with the dynamics of the system. One of the main reasons that we had more tendency towards vision is that it does not interfere with the oscillations of the metronome rods or their synchronization.
} 


\section{Adaptation}

In this research we showed some cases and considered the evolution of the system with the relevant parameters and initial conditions. A possible area to be investigated is studying the space of parameters and the evolution of the system for different regions, i.e. what is the role of initial conditions and parameters of the system (such as behaviors of the cart) on the evolution of the system. 


\section{REFERENCES}

[1] A. Andronov and S. Khaikin, Theory of Oscillations (in Russian). Moscow: Gostekhizdat, 1937.

[2] A. Andronov, A. Vitt, and S. Khaikin, Theory of Oscillations. Mineola, NY: Courier Dover Publications, 1987.

[3] E. Appleton, "The automatic synchronization of triode oscillators," Proc. Cambridge Philos. Soc. (Math. and Phys. Sci.), vol. 21, pp. 231-248, 1922.

[4] B. van der Pol, "A theory of the amplitude of free and forced triode vibrations," Radio Review, vol. 1, pp. 701-710, 754-762, 1920.

[5] B. van der Pol, "Forced oscillations in a circuit with non-linear resistance (reception with reactive triode)," The London, Edinburgh, and Dublin Philosophical Magazine and Journal of Science Ser.7, vol. 3, pp. 65-80, 1927.

[6] A. Pikovsky, M. Rosenblum, and J. Kurths, Synchronization: A Universal Concept in Nonlinear Science. Cambridge, UK: Cambridge University Press, Jan 2002.

[7] R. J. S. Rayleigh, The Theory of Sound, vol. 2. London, UK: MacMillan, 1896.

[8] M. Abel, K. Ahnert, and S. Bergweiler, "Synchronization of sound sources," Phys. Rev. Lett., vol. 103, p. 114301, Sep 2009.

[9] S. H. Strogatz, Nonlinear Dynamics and Chaos: With Applications to Physics, Biology, Chemistry, and Engineering. Studies in Non-Linearity, Cambridge, MA: Westview Press, Jan 2001. 
[10] S. H. Strogatz, Sync: The Emerging Science of Spontaneous Order. New York, NY: Hyperion Press, 2003.

[11] H. Fujisaka and T. Yamada, "Stability theory of synchronized motion in coupledoscillator systems," Progress of Theoretical Physics, vol. 69, no. 1, pp. 32-47, 1983.

[12] L. M. Pecora and T. L. Carroll, "Synchronization in chaotic systems," Phys. Rev. Lett., vol. 64, pp. 821-824, Feb 1990.

[13] L. M. Pecora and T. L. Carroll, "Driving systems with chaotic signals," Phys. Rev. A, vol. 44, pp. 2374-2383, Aug 1991.

[14] M. de Sousa Vieira, A. J. Lichtenberg, and M. A. Lieberman, "Synchronization of regular and chaotic systems," Phys. Rev. A, vol. 46, pp. R7359-R7362, Dec 1992.

[15] M. Senator, "Synchronization of two coupled escapement-driven pendulum clocks," Journal of Sound and Vibration, vol. 291, no. 3-5, pp. 566-603, 2006.

[16] I. I. Blekhman, Synchronization in Science and Technology. New York: ASME Press, 1988.

[17] M. Bennett, M. F. Schatz, H. Rockwood, and K. Wiesenfeld, "Huygens's clocks," Proceedings of the Royal Society of London. Series A: Mathematical, Physical and Engineering Sciences, vol. 458, no. 2019, pp. 563-579, 2002.

[18] W. H. Eccles, "Method of producing compound radiation fields by multiple antennae systems and the employment of a master oscillator." British Patent 184282 , May 1921. 
[19] W. H. Eccles, "Studies from a wireless laboratory," Electrician, vol. 89, no. 503, pp. 195-214, 1923.

[20] Z. Néda, E. Ravasz, Y. Brechet, T. Vicsek, and A.-L. Barabási, "Self-organizing processes: The sound of many hands clapping," Nature, vol. 403, pp. 849-850, Feb 2000.

[21] M. Rosenblum and A. Pikovsky, "Synchronization: from pendulum clocks to chaotic lasers and chemical oscillators," Contemporary Physics, vol. 44, no. 5, pp. 401-416, 2003.

[22] N. Kuznetsov, G. Leonov, H. Nijmeijer, and A. Pogromski, "Synchronization of two metronomes," in Third IFAC Workshop, (St. Petersburg, Russian Federation), pp. 1-4, Aug 2007.

[23] J. Pantaleone, "Synchronization of metronomes," American Journal of Physics, vol. 70, no. 10, pp. 992-1000, 2002.

[24] K. Czolczynski, P. Perlikowski, A. Stefanski, and T. Kapitaniak, "Clustering and synchronization of n huygens' clocks," Physica A: Statistical Mechanics and its Applications, vol. 388, no. 24, pp. 5013-5023, 2009.

[25] H. Ulrichs, A. Mann, and U. Parlitz, "Synchronization and chaotic dynamics of coupled mechanical metronomes," Chaos: An Interdisciplinary Journal of Nonlinear Science, vol. 19, no. 4, p. 043120, 2009.

[26] P. Dallard, A. J. Fitzpatrick, A. Flint, S. L. Bourva, A. Low, R. M. R. Smith, and M. Wilford, "The London millennium footbridge," Structural Engineer, vol. 79, pp. 17-35, Nov 2001. 
[27] S. H. Strogatz, D. M. Abrams, A. McRobie, B. Eckhardt, and E. Ott, "Crowd synchrony on the millennium bridge," Nature, vol. 438, pp. 43-44, Nov 2005.

[28] A. Y. Pogromsky, "Synchronization and adaptive synchronization in semi-passive systems," in 1st International Conference on Control of Oscillations and Chaos, vol. 1, (St. Petersburg, Russia), pp. 64-68, Aug 1997.

[29] I. I. Blekhman, A. L. Fradkov, H. Nijmeijer, and A. Y. Pogromsky, "On selfsynchronization and controlled synchronization," Syst. Control Lett., vol. 31, no. 5, pp. 299-305, 1997.

[30] S. J. Hodge, M. B. V. Bell, and M. A. Cant, "Reproductive competition and the evolution of extreme birth synchrony in a cooperative mammal." Published online before print in Biology letters http://bit.ly/gZ2ZTR, 2010.

[31] M. Chen and J. Kurths, "Synchronization of time-delayed systems," Phys. Rev. E, vol. 76, p. 036212, Sep 2007.

[32] A. Pikovsky, M. Rosenblum, and J. Kurths, "Phase synchronization in regular and chaotic systems," Int. J. Bifurcation Chaos, vol. 10, no. 10, pp. 2291-2305, 2000 .

[33] M. Barahona and L. M. Pecora, "Synchronization in small-world systems," Physical Review Letters, vol. 89, no. 5, p. 054101, 2002.

[34] M. Chavez, D. U. Hwang, A. Amann, H. G. Hentschel, and S. Boccaletti, "Synchronization is enhanced in weighted complex networks," Phys Rev Lett, vol. 94, p. 218701, Jun 2005.

[35] R. Tonjes, N. Masuda, and H. Kori, "Synchronization transition of identical phase oscillators in a directed small-world network," Chaos: An Interdisciplinary 
Journal of Nonlinear Science, vol. 20, no. 3, p. 033108, 2010.

[36] X. B. Lu, X. F. Wang, X. Li, and J. Q. Fang, "Synchronization in weighted complex networks: Heterogeneity and synchronizability," Physica A: Statistical Mechanics and its Applications, vol. 370, no. 2, pp. 381-389, 2006.

[37] M. Chavez, D.-U. Hwang, A. Amann, and S. Boccaletti, "Synchronizing weighted complex networks," Chaos: An Interdisciplinary Journal of Nonlinear Science, vol. 16, no. 1, p. 015106, 2006.

[38] M. Chavez, D.-U. Hwang, J. Martinerie, and S. Boccaletti, "Degree mixing and the enhancement of synchronization in complex weighted networks," Phys. Rev. E, vol. 74, p. 066107, Dec 2006.

[39] S. Boccaletti, D.-U. Hwang, M. Chavez, A. Amann, J. Kurths, and L. M. Pecora, "Synchronization in dynamical networks: Evolution along commutative graphs," Phys. Rev. E, vol. 74, p. 016102, Jul 2006.

[40] M. G. Rosenblum, A. S. Pikovsky, and J. Kurths, "Phase synchronization of chaotic oscillators," Physical Review Letters, vol. 76, pp. 1804-1807, Mar 1996.

[41] F. Mormann, T. Kreuz, R. G. Andrzejak, P. David, K. Lehnertz, and C. E. Elger, "Epileptic seizures are preceded by a decrease in synchronization.," Epilepsy Res, vol. 53, pp. 173-185, Mar 2003.

[42] K. Lehnertz, S. Bialonski, M.-T. Horstmann, D. Krug, A. Rothkegel, M. Staniek, and T. Wagner, "Synchronization phenomena in human epileptic brain networks," Journal of Neuroscience Methods, vol. 183, no. 1, pp. 42-48, 2009.

[43] M. Mackey and L. Glass, "Oscillation and chaos in physiological control systems," Science, vol. 197, no. 4300, pp. 287-289, 1977. 
[44] J. D. Farmer, "Chaotic attractors of an infinite-dimensional dynamical system," Physica D: Nonlinear Phenomena, vol. 4, no. 3, pp. 366-393, 1982.

[45] B. Dorizzi, B. Grammaticos, M. Le Berre, Y. Pomeau, E. Ressayre, and A. Tallet, "Statistics and dimension of chaos in differential delay systems," Phys. Rev. A, vol. 35, pp. 328-339, Jan 1987.

[46] S. Yanchuk and P. Perlikowski, "Delay and periodicity," Phys. Rev. E, vol. 79, p. 046221, Apr 2009.

[47] H. U. Voss, "Anticipating chaotic synchronization," Physical Review E, vol. 61, pp. 5115-5119, May 2000.

[48] N. H. Packard, "Adaptation toward the edge of chaos," in Dynamic Patterns in Complex Systems (A. K. AJ Mandell and M. Shlesinger, eds.), (Singapore), pp. 293-301, World Scientific Pub Co Inc, Jan 1988.

[49] M. Mitchell, P. T. Hraber, and J. P. Crutchfield, "Revisiting the edge of chaos: Evolving cellular automata to perform computations," Complex Systems, vol. 7, pp. 89-130, 1993.

[50] S. A. Kauffman and S. Johnsen, "Coevolution to the edge of chaos: Coupled fitness landscapes, poised states, and coevolutionary avalanches," Journal of Theoretical Biology, vol. 149, no. 4, pp. 467-505, 1991.

[51] D. Pierre and A. Hübler, "A theory for adaptation and competition applied to logistic map dynamics," Physica D: Nonlinear Phenomena, vol. 75, no. 1-3, pp. 343-360, 1994.

[52] A. W. Hübler and T. Wotherspoon, "Self-adjusting systems avoid chaos," Complexity, vol. 14, no. 4, pp. 8-11, 2009. 
[53] P. Melby, J. Kaidel, N. Weber, and A. Hübler, "Adaptation to the edge of chaos in the self-adjusting logistic map," Physical Review Letters, vol. 84, pp. 59915993, Jun 2000.

[54] L. Chua, M. Komuro, and T. Matsumoto, "The double scroll family," IEEE Transactions on Circuits and Systems, vol. 33, pp. 1072-1118, Nov 1986.

[55] M. Baym and A. W. Hübler, "Conserved quantities and adaptation to the edge of chaos," Phys. Rev. E, vol. 73, pp. 056210-056217, May 2006.

[56] C. G. Langton, "Computation at the edge of chaos: phase transitions and emergent computation," Phys. D, vol. 42, no. 1-3, pp. 12-37, 1990.

[57] J. P. Crutchfield and K. Young, "Computation at the onset of chaos," in Entropy, Complexity, and the Physics of Information (W. Zurek, ed.), SFI Studies in the Sciences of Complexity, VIII, pp. 223-269, Reading, MA: Addison-Wesley, 1990.

[58] A. W. Hübler and K. C. Phelps, "Guiding an adaptive system through chaos," Complexity, vol. 13, no. 2, pp. 62-66, 2007.

[59] S. A. Kauffman, The Origins of Order: Self-Organization and Selection in Evolution. New York, NY: Oxford University Press, 1993.

[60] N. Bertschinger and T. Natschläger, "Real-time computation at the edge of chaos in recurrent neural networks," Neural Comput., vol. 16, pp. 1413-1436, Jul 2004.

[61] F. Schürmann, K. Meier, and J. Schemmel, "Edge of chaos computation in mixed-mode vlsi - a hard liquid," in Advances in Neural Information Processing Systems 17 (L. K. Saul, Y. Weiss, and L. Bottou, eds.), pp. 1201-1208, Cambridge, MA: MIT Press, 2005. 
[62] M. Mitchell, J. P. Crutchfield, and P. T. Hraber, Dynamics, Computation, and the "Edge of Chaos": a Re-Examination, pp. 497-513. Cambridge, MA, USA: Perseus Books, 1999.

[63] J. A. Bryan, "Investigating the conservation of mechanical energy using video analysis: four cases," Physics Education, vol. 45, no. 1, pp. 50-57, 2010.

[64] D. Aubert and C. Thorpe, "Color image processing for navigation: Two road trackers," Tech. Rep. CMU-RI-TR-90-09, Robotics Institute, Pittsburgh, PA, Apr 1990.

[65] W. T. Oud, H. Nijmeijer, and A. Y. Pogromsky, "A study of huijgens synchronization: Experimental results," in Group Coordination and Cooperative Control (K. Pettersen, J. Gravdahl, and H. Nijmeijer, eds.), vol. 336 of Lecture Notes in Control and Information Sciences, pp. 191-203, Berlin: Springer, 2006. 


\section{VITA}

Roozbeh Daneshvar received his Bachelor of Science degree in computer engineering (software engineering) from University of Tehran, Tehran, Iran in 2002. He received his Master of Science in computer engineering (artificial intelligence and robotics) in 2005 . His research interests include complex systems, non-linear dynamics, synchronization, chaos, self organization and emergence.

Mr. Daneshvar may be reached at:

H.R. Bright Building, Rm. 701, Ross Street

TAMU 3141, College Station TX 77843-3141.

His webpage URL is http://www.daneshvar.ir/roozbeh and his email address is roozbeh@tamu.edu. 